\title{
Multivalued Fixed Point Results for New Generalized F-Dominated Contractive Mappings on Dislocated Metric Space with Application
}

\author{
Tahair Rasham ${ }^{D},{ }^{1}$ Abdullah Shoaib $\left(\mathbb{D},{ }^{2}\right.$ Badriah A. S. Alamri, ${ }^{3}$ and Muhammad Arshad \\ ${ }^{1}$ Department of Mathematics, International Islamic University, H-10, Islamabad 44000, Pakistan \\ ${ }^{2}$ Department of Mathematics and Statistics, Riphah International University, Islamabad 44000, Pakistan \\ ${ }^{3}$ Department of Mathematics, King Abdulaziz University, P.O. Box 80203, Jeddah 21589, Saudi Arabia
}

Correspondence should be addressed to Tahair Rasham; tahir_resham@yahoo.com

Received 19 October 2017; Accepted 27 November 2017; Published 1 February 2018

Academic Editor: Manuel De la Sen

Copyright (c) 2018 Tahair Rasham et al. This is an open access article distributed under the Creative Commons Attribution License, which permits unrestricted use, distribution, and reproduction in any medium, provided the original work is properly cited.

The purpose of this paper is to find out fixed point results for semi- $\alpha_{*}$-dominated multivalued mappings fulfilling a new generalized locally $F$-dominated multivalued contractive condition on a closed ball in complete dislocated metric space. Example and application both are given to show the novelty of our results. Our results merge, extend, and infer many results.

\section{Introduction and Preliminaries}

Fixed point theory has a foundational role in functional analysis. Banach [1] established the fundamental fixed point theorem, which has played a significant role in different fields of applied mathematics. Due to its significance, a large number of authors have proven many interesting multiplications of his result (see [1-33]). Many authors introduced fixed point theorems in complete dislocated metric space. The idea of dislocated topology has been applied in the field of logic programming semantics (see [14]).

Wardowski [33] introduced new type of contraction called $F$-contraction and showed a new generalized fixed point theorem. He generalized many previous fixed point results in different directions. Many other useful results on $F$-contractions can be seen in $[3-5,11,12,15,18,22,24-27]$. In this paper, we recalled the concept of $F$-contraction to obtain some common fixed point results for semi- $\alpha_{*}$-dominated multivalued mappings on proximinal sets satisfying a new type rational $F$-contraction in the context of complete dislocated metric spaces. We have also given an example in which the mapping is not $\alpha_{*}$-admissible but it fulfills the condition of $\alpha_{*}$-dominated.
Definition 1 (see [14]). Let $Z$ be a nonempty set. A mapping

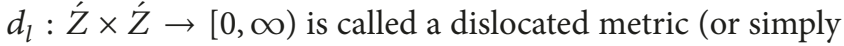
$d_{l}$-metric) if the following conditions hold, for any $\check{t}, \check{s}, \check{z} \in \dot{Z}$ :

(i) If $d_{l}(\check{t}, \check{s})=0$, then $\check{t}=\check{s}$.

(ii) $d_{l}(\check{t}, \check{s})=d_{l}(\check{s}, \check{t})$.

(iii) $d_{l}(\check{t}, \check{s}) \leq d_{l}(\check{t}, \check{z})+d_{l}(\check{z}, \check{s})$.

Then $d_{l}$ is called a dislocated metric on $\dot{Z}$, and the pair $\left(Z, d_{l}\right)$ is called dislocated metric space or $d_{l}$ metric space. It is clear that if $d_{l}(\check{t}, \check{s})=0$, then, from (i), $\check{t}=\check{s}$. But if $\check{t}=\check{s}$, $d_{l}(\check{t}, \check{s})$ may not be 0 . We use DMS instead of dislocated metric space.

Definition 2 (see [14]). Let $\left(Z, d_{l}\right)$ be a DMS:

(i) A sequence $\left\{c_{r}\right\}$ in $\left(Z, d_{l}\right)$ is called a Cauchy sequence if given $\varepsilon>0$; there corresponds $\check{n}_{0} \in N$ such that for all $\check{n}, m \geq \check{n}_{0}$ one has $d_{l}\left(c_{m}, c_{\breve{n}}\right)<\varepsilon$ or $\lim _{\check{n}, m \rightarrow \infty} d_{l}\left(c_{\check{n}}, c_{m}\right)=0$.

(ii) A sequence $\left\{c_{\hat{n}}\right\}$ is said to be dislocated-converges (for short $d_{l}$-converges) to $l$ if $\lim _{\check{n} \rightarrow \infty} d_{l}\left(c_{\breve{n}}, l\right)=0$. In this case $l$ is called a $d_{l}$-limit of $\left\{c_{\tilde{n}}\right\}$. 
(iii) $\left(\dot{Z}, d_{l}\right)$ is called complete if every Cauchy sequence in $\dot{Z}$ converges to a point $l \in \dot{Z}$ such that $d_{l}(l, l)=0$.

Definition 3 (see [29]). Let $H$ be a nonempty subset of DMS $\dot{Z}$ and let $i \in \dot{Z}$. An element $v_{0} \in H$ is called a best approximation in $H$ if

$$
d_{l}(i, H)=d_{l}\left(i, v_{0}\right), \quad \text { where } d_{l}(i, H)=\inf _{y \in H} d_{l}(i, y) .
$$

If each $i \in \dot{Z}$ has at least one best approximation in $H$, then $H$ is called a proximinal set. We denote $P(Z)$ as the set of all closed proximinal subsets of $\dot{Z}$.

Definition 4 (see [29]). The function $H_{d_{l}}: P(\dot{Z}) \times P(\dot{Z}) \rightarrow R^{+}$, defined by

$$
H_{d_{l}}(N, M)=\max \left\{\sup _{\check{n} \in N} d_{l}(\check{n}, M), \sup _{m \in M} d_{l}(N, m)\right\},
$$

is called dislocated Hausdorff metric on $P(Z)$.

Definition 5 (see [29]). Let $\check{S}: \check{Z} \rightarrow P(\dot{Z})$ be a multivalued mapping and $\alpha: \dot{Z} \times \dot{Z} \rightarrow[0,+\infty)$. Let $K \subseteq \dot{Z}$; we say that $\check{S}$ is semi- $\alpha^{\star}$-admissible on $K$, whenever $\alpha(i, j) \geq 1$ implies that $\alpha^{\star}(\check{S} i, \check{S} j) \geq 1$, for all $i, j \in K$, where $\alpha^{\star}(\check{S} i, \check{S} j)=\inf \{\alpha(u, v)$ : $u \in \check{S} i, v \in \check{S} j$ \}. If $K=\check{Z}$, then we say that $\check{S}$ is $\alpha^{\star}$-admissible on $\dot{Z}$.

Definition 6. Let $\left(\dot{Z}, d_{l}\right)$ be a DMS. Let $\check{S}: \dot{Z} \rightarrow P(\dot{Z})$ be multivalued mapping and $\alpha: \dot{Z} \times \dot{Z} \rightarrow[0,+\infty)$. Let $A \subseteq$ $\dot{Z}$, and we say that $\check{S}$ is semi- $\alpha_{*}$-dominated on $H$, whenever $\alpha_{*}(i, \check{S} i) \geq 1$ for all $i \in H$, where $\alpha_{*}(i, \check{S} i)=\inf \{\alpha(i, l): l \in$ $\check{S} i\}$. If $H=\dot{Z}$, then we say that $\check{S}$ is $\alpha_{*}$-dominated on $\dot{Z}$. If $\check{S}: Z^{\prime} \rightarrow Z^{\prime}$ is a self-mapping, then $\check{S}$ is semi- $\alpha$-dominated on $H$, whenever $\alpha(i, \check{S} i) \geq 1$ for all $i \in H$.

Definition 7 (see [33]). Let $(Z, d)$ be a metric space. A mapping $H: Z \rightarrow Z$ is said to be an $F$-contraction if there exists $\tau>0$ such that

$$
\begin{gathered}
d(H j, H k)>0 \Longrightarrow \\
\tau+F(d(H j, H k)) \leq F(d(j, k))
\end{gathered}
$$

$$
\forall j, k \in \dot{Z},
$$

where $F: \mathbb{R}_{+} \rightarrow \mathbb{R}$ is a mapping satisfying the following conditions:

(F1) $F$ is strictly increasing; that is, for all $j, k \in \mathbb{R}_{+}$, such that $j<k, F(j)<F(k)$.

(F2) For each sequence $\left\{\alpha_{\check{n}}\right\}_{\check{n}=1}^{\infty}$ of positive numbers, $\lim _{\check{n} \rightarrow \infty} \alpha_{\check{n}}=0$ if and only if $\lim _{\check{n} \rightarrow \infty} F\left(\alpha_{\check{n}}\right)=-\infty$. 0 .

(F3) There exists $k \in(0,1)$ such that $\lim \alpha \rightarrow 0^{+} \alpha^{k} F(\alpha)=$

Lemma 8. Let $\left(\dot{Z}, d_{l}\right)$ be a DMS. Let $\left(P(\dot{Z}), H_{d_{l}}\right)$ be a dislocated Hausdorff metric space on $P(\dot{Z})$. Then, for all $G, H \in$
$P(\dot{Z})$ and for each $g \in G$, there exists $h_{g} \in H$ satisfying $d_{l}(g, H)=d_{l}\left(g, h_{g}\right)$; then $H_{d_{l}}(G, H) \geq d_{l}\left(g, h_{g}\right)$.

Example 9 (see [14]). If $\dot{Z}=R^{+} \cup\{0\}$, then $d_{l}(j, k)=j+k$ defines a dislocated metric $d_{l}$ on $\dot{Z}$.

Example 10 (see [33]). The family of $F$ is not empty:

(1) $F(\check{c})=\ln (\check{c}) ; \check{c}>0$.

(2) $F(\check{c})=\check{c}+\ln (\check{c}) ; \check{c}>0$.

(3) $F(\check{c})=-1 / \sqrt{\check{c}} ; \check{c}>0$.

Example 11. Let $\dot{Z}=\mathbb{R}$. Define the mapping $\alpha: \dot{Z} \times \dot{Z} \rightarrow$ $[0, \infty)$ by

$$
\alpha(j, k)= \begin{cases}1 & \text { if } j>k \\ \frac{1}{2} & \text { otherwise }\end{cases}
$$

Define the multivalued mappings $\check{S}, \check{T}: \check{Z} \rightarrow P(\dot{Z})$ by

$$
\begin{aligned}
& \check{S} j=\{[j-4, j-3] \text { if } j \in \check{Z}\}, \\
& \check{T} k=\{[k-2, k-1] \text { if } k \in \dot{Z}\} .
\end{aligned}
$$

Suppose that $j=3$ and $k=2.5$. As $3>2.5$, then $\alpha(3,2.5) \geq 1$. Now, $\alpha_{\star}(\check{S} 3, \check{T} 2.5)=\inf \{\alpha(a, b): a \in \check{S} 3, b \in \check{T} 2.5\}=1 / 2 \nsupseteq$ 1 , and this means that the pair $(\check{S}, \check{T})$ is not $\alpha_{\star}$-admissible. Also, $\alpha_{\star}(\check{S} 3, \check{S} 2) \geq 1$ and $\alpha_{\star}(\check{T} 3, \check{T} 2) \geq 1$. This implies that $\check{S}$ and $\check{T}$ are not $\alpha_{\star}$-admissible individually. Now, $\alpha_{\star}(j, \check{S} j)=$ $\inf \{\alpha(j, b): b \in \check{S} j\} \geq 1$, for all $j \in \check{Z}$. Hence $\check{S}$ is $\alpha_{\star}-$ dominated mapping. Similarly, $\alpha_{\star}(k, \check{T} k)=\inf \{\alpha(k, b): b \in$ $\check{T} k\} \geq 1$. Hence it is clear that $\check{S}$ and $\check{T}$ are $\alpha_{\star}$-dominated but not $\alpha_{\star}$-admissible.

\section{Main Result}

Let $\left(\dot{Z}, d_{l}\right)$ be a DMS, let $c_{0} \in \dot{Z}$, and let $\check{S}$, $\check{T}: \dot{Z} \rightarrow P(\dot{Z})$ be the multifunctions on $\dot{Z}$. Let $c_{1} \in \check{S} c_{0}$ be an element such that $d_{l}\left(c_{0}, \check{S} c_{0}\right)=d_{l}\left(c_{0}, c_{1}\right)$. Let $c_{2} \in \check{T} c_{1}$ be such that $d_{l}\left(c_{1}, \check{T} c_{1}\right)=d_{l}\left(c_{1}, c_{2}\right)$. Let $c_{3} \in \check{S} c_{2}$ be such that $d_{l}\left(c_{2}, \check{S} c_{2}\right)=$ $d_{l}\left(c_{2}, c_{3}\right)$. Continuing this method, we get a sequence $c_{\check{n}}$ of points in $\dot{Z}$ such that $c_{2 \check{n}+1} \in \check{S}_{2 \check{n}}$ and $c_{2 \check{n}+2} \in \check{T}_{c_{\check{n}+1}}$, where $\check{n}=0,1,2, \ldots$. Also $d_{l}\left(c_{2 \check{n}}, \check{S}_{2 \check{n}}\right)=d_{l}\left(c_{2 \check{n}}, c_{2 \check{n}+1}\right)$, and $d_{l}\left(c_{2 \check{n}+1}, \check{T} c_{2 \check{n}+1}\right)=d_{l}\left(c_{2 \check{n}+1}, c_{2 \check{n}+2}\right)$. We denote this iterative sequence by $\left\{\check{T} \check{S}\left(c_{\check{n}}\right)\right\}$. We say that $\left\{\check{T} \check{S}\left(c_{\check{n}}\right)\right\}$ is a sequence in Ź generated by $c_{0}$.

Theorem 12. Let $\left(\dot{Z}, d_{l}\right)$ be a complete DMS. Suppose that there exists a function $\alpha: \dot{Z} \times \dot{Z} \rightarrow[0, \infty)$. Let $\check{r}>0$, 
$c_{0} \in \overline{B_{d_{l}}\left(c_{0}, r\right)} \subseteq \dot{Z}$ and let $\check{S}, \check{T}: \check{Z} \rightarrow P(\dot{Z})$ be the semi$\alpha_{*}$-dominated mappings on $\overline{B_{d_{1}}\left(c_{0}, \check{r}\right)}$. Assume that, for some $\tau>0$,

$$
\begin{aligned}
& \max \left\{\tau+\alpha_{*}(\breve{e}, \check{S} \breve{e}) F\left(H_{d_{l}}(\check{S} \breve{e}, \check{T} \hat{y})\right), \tau\right. \\
& \left.+\alpha_{*}(\hat{y}, \check{T} \hat{y}) F\left(H_{d_{l}}(\check{T} \hat{y}, \check{S} \breve{e})\right)\right\} \leq F\left(\eta_{1} d_{l}(\breve{e}, \widehat{y})\right. \\
& +\eta_{2} d_{l}(\breve{e}, \check{S} \breve{e})+\eta_{3} d_{l}(\breve{e}, \check{T} \hat{y}) \\
& \left.+\eta_{4} \frac{d_{l}^{2}(\breve{e}, \check{S} \breve{e}) \cdot d_{l}(\hat{y}, \check{T} \hat{y})}{1+d_{l}^{2}(\breve{e}, \widehat{y})}\right)
\end{aligned}
$$

for all $\breve{e}, \hat{y} \in \overline{B_{d_{1}}\left(c_{0}, \check{r}\right)} \cap\left\{\check{T} \check{S}\left(c_{\check{n}}\right)\right\}$ with either $\alpha(\breve{e}, \hat{y}) \geq 1$ or $\alpha(\hat{y}, \breve{e}) \geq 1$, where $\eta_{1}, \eta_{2}, \eta_{3}, \eta_{4}>0$ and $\eta_{1}+\eta_{2}+2 \eta_{3}+\eta_{4}<1$ and

$$
d_{l}\left(c_{0}, \check{S} c_{0}\right) \leq(1-\lambda) \check{r}
$$

where $\lambda=\left(\left(\eta_{1}+\eta_{2}+\eta_{3}\right) /\left(1-\eta_{3}-\eta_{4}\right)\right)$ and $\eta_{3}+\eta_{4} \neq 1$. Then $\left\{\check{T} \check{S}\left(c_{\check{n}}\right)\right\}$ is a sequence in $\overline{B_{d_{1}}\left(c_{0}, \check{r}\right)}, \alpha\left(c_{\check{n}}, c_{\check{n}+1}\right) \geq 1$ for all $\check{n} \in N \cup\{0\}$, and $\left\{\check{T} \check{S}\left(c_{\breve{n}}\right)\right\} \rightarrow \bar{u} \in \overline{B_{d_{1}}\left(c_{0}, \check{r}\right)}$. Also if inequality (6) holds for $\bar{u}$ and either $\alpha\left(c_{\breve{n}}, \bar{u}\right) \geq 1$ or $\alpha\left(\bar{u}, c_{\check{n}}\right) \geq 1$ for all $\check{n} \epsilon$ $N \cup\{0\}$, then $\check{S}$ and $\check{T}$ have common fixed point $\bar{u}$ in $\overline{B_{d_{l}}\left(c_{0}, \check{r}\right)}$.

Proof. Consider a sequence $\left\{\check{T} \check{S}\left(c_{\check{n}}\right)\right\}$. From (7), we get

$$
d_{l}\left(c_{0}, c_{1}\right) \leq d_{l}\left(c_{0}, \check{S} c_{0}\right) \leq \check{r} .
$$

It follows that

$$
c_{1} \in \overline{B_{d_{l}}\left(c_{0}, \check{r}\right)} .
$$

Let $c_{2}, \ldots, c_{j} \in \overline{B_{d_{l}}\left(c_{0}, \check{r}\right)}$ for some $j \in N$. If $j=2 \grave{i}+1$, where $\grave{i}=1,2, \ldots,(j-1) / 2$, then since $\check{S}, \check{T}: \dot{Z} \rightarrow P(\dot{Z})$ are semi- $\alpha_{*}$-dominated mappings on $\overline{B_{d_{l}}\left(c_{0}, \check{r}\right)}, \alpha_{*}\left(c_{2 i}, \check{S} c_{2 i}\right) \geq 1$ and $\alpha_{*}\left(c_{2 i+1}, \check{T} c_{2 i+1}\right) \geq 1$. As $\alpha_{*}\left(c_{2 i}, \check{S} c_{2 i}\right) \geq 1$, this implies that $\inf \left\{\alpha\left(c_{2 i}, b\right): b \in \check{S}_{c_{i i}}\right\} \geq 1$. Also $c_{2 i+1} \in \check{S}_{c_{2 i}}$, so $\alpha\left(c_{2 i}, c_{2 i+1}\right) \geq$ 1 . Now, by using Lemma 8 , we have

$$
\begin{aligned}
\tau+ & F\left(d_{l}\left(c_{2 i+1}, c_{2 i+2}\right)\right) \leq \tau+F\left(H_{d_{l}}\left(\check{S} c_{2 i}, \check{T} c_{2 i+1}\right)\right) \\
& \leq \max \left\{\tau+\alpha_{*}\left(c_{2 i}, \check{S} c_{2 i}\right) F\left(H_{d_{l}}\left(\check{S} c_{2 i}, \check{T} c_{2 i+1}\right)\right), \tau\right. \\
& \left.+\alpha_{*}\left(c_{2 i+1}, \check{T} c_{2 i+1}\right) F\left(H_{d_{l}}\left(\check{T} c_{2 i+1}, \check{S} c_{2 i}\right)\right)\right\} \\
\leq & F\left[\eta_{1} d_{l}\left(c_{2 i}, c_{2 i+1}\right)+\eta_{2} d_{l}\left(c_{2 i}, \check{S} c_{2 i}\right)\right. \\
& +\eta_{3} d_{l}\left(c_{2 i}, \check{T} c_{2 i+1}\right) \\
& \left.+\eta_{4} \frac{d_{l}^{2}\left(c_{2 i}, \check{S} c_{2 i}\right) \cdot d_{l}\left(c_{2 i+1}, \check{T} c_{2 i+1}\right)}{1+d_{l}^{2}\left(c_{2 i}, c_{2 i+1}\right)}\right]
\end{aligned}
$$

$$
\begin{aligned}
\leq & F\left[\eta_{1} d_{l}\left(c_{2 i}, c_{2 i+1}\right)+\eta_{2} d_{l}\left(c_{2 i}, c_{2 i+1}\right)\right. \\
& +\eta_{3} d_{l}\left(c_{2 i}, c_{2 i+1}\right)+\eta_{3} d_{l}\left(c_{2 i+1}, c_{2 i+2}\right) \\
& \left.+\eta_{4} \frac{d_{l}^{2}\left(c_{2 i}, c_{2 i+1}\right) \cdot d_{l}\left(c_{2 i+1}, c_{2 i+2}\right)}{1+d_{l}^{2}\left(c_{2 i}, c_{2 i+1}\right)}\right] \\
\leq & F\left(\left(\eta_{1}+\eta_{2}+\eta_{3}\right) d_{l}\left(c_{2 i}, c_{2 i+1}\right)+\left(\eta_{3}\right.\right. \\
& \left.\left.+\eta_{4}\right) d_{l}\left(c_{2 i+1}, c_{2 i+2}\right)\right)-\tau ;
\end{aligned}
$$

this implies that

$$
\begin{aligned}
& F\left(d_{l}\left(c_{2 i+1}, c_{2 i+2}\right)\right) \leq F\left(\left(\eta_{1}+\eta_{2}+\eta_{3}\right) d_{l}\left(c_{2 i}, c_{2 i+1}\right)\right. \\
& \left.\quad+\left(\eta_{3}+\eta_{4}\right) d_{l}\left(c_{2 i+1}, c_{2 i+2}\right)\right),
\end{aligned}
$$

for all $j \in N$. As $F$ is strictly increasing, we have

$$
\begin{aligned}
d_{l}\left(c_{2 i+1}, c_{2 i+2}\right)< & \left(\eta_{1}+\eta_{2}+\eta_{3}\right) d_{l}\left(c_{2 i}, c_{2 i+1}\right) \\
& +\left(\eta_{3}+\eta_{4}\right) d_{l}\left(c_{2 i+1}, c_{2 i+2}\right),
\end{aligned}
$$

which implies that

$$
\begin{aligned}
& \left(1-\eta_{3}-\eta_{4}\right) d_{l}\left(c_{2 i+1}, c_{2 i+2}\right) \\
& <\left(\eta_{1}+\eta_{2}+\eta_{3}\right) d_{l}\left(c_{2 i}, c_{2 i+1}\right) \\
& d_{l}\left(c_{2 i+1}, c_{2 i+2}\right)<\left(\frac{\eta_{1}+\eta_{2}+\eta_{3}}{1-\eta_{3}-\eta_{4}}\right) d_{l}\left(c_{2 i}, c_{2 i+1}\right) .
\end{aligned}
$$

Here $\lambda=\left(\left(\eta_{1}+\eta_{2}+\eta_{3}\right) /\left(1-\eta_{3}-\eta_{4}\right)\right)<1$. Hence

$$
\begin{aligned}
d_{l}\left(c_{2 i+1}, c_{2 i+2}\right) & <\lambda d_{l}\left(c_{2 i}, c_{2 i+1}\right)<\lambda^{2} d_{l}\left(c_{2 i-1}, c_{2 i}\right)<\cdots \\
& <\lambda^{j} d_{l}\left(c_{0}, c_{1}\right) .
\end{aligned}
$$

Now,

$$
\begin{aligned}
d_{l}\left(c_{0}, c_{j+1}\right) & \leq d_{l}\left(c_{0}, c_{1}\right)+d_{l}\left(c_{1}, c_{2}\right)+\cdots+d_{l}\left(c_{j}, c_{j+1}\right) \\
& \leq d_{l}\left(c_{0}, c_{1}\right)\left[1+\lambda+\cdots+\lambda^{j}\right] \\
& \leq(1-\lambda) \check{r} \frac{\left(1-\lambda^{j+1}\right)}{(1-\lambda)}<\check{r} .
\end{aligned}
$$


Thus, $c_{j+1} \in \overline{B_{d_{l}}\left(c_{0}, \check{r}\right)}$. Hence $c_{\check{n}} \in \overline{B_{d_{l}}\left(c_{0}, \check{r}\right)}$, for all $\check{n} \in N$. Continuing this process, we get

$$
\begin{aligned}
\tau+ & F\left(d_{l}\left(c_{\check{n}}, c_{\check{n}+1}\right)\right) \leq \tau+F\left(H_{d_{l}}\left(\check{S} c_{\check{n}-1}, \check{T} c_{\check{n}}\right)\right) \\
& \leq \max \left\{\tau+\alpha_{*}\left(c_{\check{n}-1}, \check{S} c_{\check{n}-1}\right) F\left(H_{d_{l}}\left(\check{S} c_{\check{n}-1}, \check{T} c_{\check{n}}\right)\right), \tau\right. \\
& \left.+\alpha_{*}\left(c_{\check{n}}, \check{T} c_{\check{n}}\right) F\left(H_{d_{l}}\left(\check{T} c_{\check{n}}, \check{S} c_{\check{n}-1}\right)\right)\right\} \\
\leq & F\left[\eta_{1} d_{l}\left(c_{\check{n}-1}, c_{\check{n}}\right)+\eta_{2} d_{l}\left(c_{\check{n}-1}, \check{S} c_{\check{n}-1}\right)\right. \\
& +\eta_{3} d_{l}\left(c_{\check{n}-1}, \check{T} c_{\check{n}}\right) \\
& \left.+\eta_{4} \frac{d_{l}^{2}\left(c_{\check{n}-1}, \check{S} c_{\check{n}-1}\right) \cdot d_{l}\left(c_{\check{n}}, \check{T} c_{\check{n}}\right)}{1+d_{l}^{2}\left(c_{\check{n}-1}, c_{\check{n}}\right)}\right] \\
\leq & F\left[\eta_{1} d_{l}\left(c_{\check{n}-1}, c_{\check{n}}\right)+\eta_{2} d_{l}\left(c_{\check{n}-1}, c_{\check{n}}\right)+\eta_{3} d_{l}\left(c_{\check{n}-1}, c_{\check{n}}\right)\right. \\
& \left.+\eta_{3} d_{l}\left(c_{\check{n}}, c_{\check{n}-1}\right)+\eta_{4} \frac{d_{l}^{2}\left(c_{\check{n}-1}, c_{\check{n}}\right) \cdot d_{l}\left(c_{\check{n}}, c_{\check{n}+1}\right)}{1+d_{l}^{2}\left(c_{\check{n}-1}, c_{\check{n}}\right)}\right] \\
\leq & F\left[\left(\eta_{1}+\eta_{2}+\eta_{3}\right) d_{l}\left(c_{\check{n}-1}, c_{\check{n}}\right)+\left(\eta_{3}\right) d_{l}\left(c_{\check{n}}, c_{\check{n}+1}\right)\right]-\tau ; \\
& {[\tau ;}
\end{aligned}
$$

this implies that

$$
\begin{aligned}
& F\left(d_{l}\left(c_{\check{n}}, c_{\check{n}+1}\right)\right) \leq F\left[\left(\eta_{1}+\eta_{2}+\eta_{3}\right) d_{l}\left(c_{\check{n}-1}, c_{\check{n}}\right)\right. \\
& \left.\quad+\left(\eta_{3}+\eta_{4}\right) d_{l}\left(c_{\check{n}}, c_{\check{n}+1}\right)\right],
\end{aligned}
$$

for all $\check{n} \in N$. As $F$ is strictly increasing, we have

$$
\begin{aligned}
d_{l}\left(c_{\check{n}}, c_{\check{n}+1}\right)< & \left(\eta_{1}+\eta_{2}+\eta_{3}\right) d_{l}\left(c_{\check{n}-1}, c_{\check{n}}\right) \\
& +\left(\eta_{3}+\eta_{4}\right) d_{l}\left(c_{\check{n}}, c_{\check{n}+1}\right),
\end{aligned}
$$

which implies that

$$
\begin{aligned}
& \left(1-\eta_{3}-\eta_{4}\right) d_{l}\left(c_{\check{n}}, c_{\check{n}+1}\right)<\left(\eta_{1}+\eta_{2}+\eta_{3}\right) d_{l}\left(c_{\check{n}-1}, c_{\check{n}}\right) \\
& d_{l}\left(c_{\check{n}}, c_{\check{n}+1}\right)<\left(\frac{\eta_{1}+\eta_{2}+\eta_{3}}{1-\eta_{3}-\eta_{4}}\right) d_{l}\left(c_{\check{n}-1}, c_{\check{n}}\right) .
\end{aligned}
$$

Here $\lambda=\left(\left(\eta_{1}+\eta_{2}+\eta_{3}\right) /\left(1-\eta_{3}-\eta_{4}\right)\right)<1$. Hence

$$
d_{l}\left(c_{\check{n}}, c_{\check{n}+1}\right)<\lambda d_{l}\left(c_{\check{n}-1}, c_{\check{n}}\right)<d_{l}\left(c_{\check{n}-1}, c_{\check{n}}\right) \text {. }
$$

Consequently,

$$
\tau+F\left(d_{l}\left(c_{\check{n}}, c_{\check{n}+1}\right)\right) \leq F\left(d_{l}\left(c_{\check{n}-1}, c_{\check{n}}\right)\right),
$$

which implies that

$$
\begin{gathered}
F\left(d_{l}\left(c_{\check{n}}, c_{\check{n}+1}\right)\right) \leq F\left(d_{l}\left(c_{\check{n}-1}, c_{\check{n}}\right)\right)-\tau \\
\vdots \\
\leq F\left(d_{1}\left(c_{0}, c_{1}\right)\right)-\check{n} \tau .
\end{gathered}
$$

This implies that

$$
F\left(d_{l}\left(c_{\check{n}}, c_{\check{n}+1}\right)\right) \leq F\left(d_{l}\left(c_{0}, c_{1}\right)\right)-\check{n} \tau
$$

And so $\lim _{\check{n} \rightarrow \infty} F\left(d_{l}\left(c_{\check{n}}, c_{\check{n}+1}\right)\right)=-\infty$. By $\left(F_{2}\right)$, we find that

$$
\lim _{\check{n} \rightarrow \infty} F\left(d_{l}\left(c_{\check{n}}, c_{\check{n}+1}\right)\right)=0
$$

We shall prove that $\left\{\check{T} \check{S}\left(c_{\check{n}}\right)\right\}$ is Cauchy in $\left(\check{Z}, d_{l}\right)$. So it suffices to show that $\lim _{\check{n} \rightarrow \infty} d_{l}\left(c_{\check{n}}, c_{m}\right)=0$. We argue by contradiction. Suppose that there exist $\epsilon>0$ and sequences $(\check{n}(q))$ and $(m(q))$ of natural numbers such that

$$
\begin{aligned}
& d_{l}\left(c_{\check{n}(q)}, c_{\check{n}(q)+1}\right) \geq \epsilon, \\
& d_{l}\left(c_{\check{n}(q)+1}, c_{m(q)}\right)<\epsilon
\end{aligned}
$$

$$
m(q)>\check{n}(q)>q, \forall q \in N \text {. }
$$

By triangular inequality, we have

$$
\begin{aligned}
d_{l}\left(c_{\check{n}(q)}, c_{m(q)}\right) & \leq d_{l}\left(c_{\check{n}(q)}, c_{\check{n}(q)+1}\right)+d_{l}\left(c_{\check{n}(q)+1}, c_{m(q)}\right) \\
& \leq \epsilon+d_{l}\left(c_{\check{n}(q)}, c_{\check{n}(q)+1}\right) \\
& \leq \epsilon+d_{l}\left(c_{\check{n}(q)}, c_{\check{n}(q)+1}\right) .
\end{aligned}
$$

From (24), there exist $q_{1} \in N$ such that, for all $q \geq q_{1}$,

$$
d_{l}\left(c_{\check{n}(q)}, \check{T} c_{\check{n}(q)}\right)<\epsilon
$$

Combining (26) with (27) yields

$$
d_{l}\left(c_{\check{n}(q)}, c_{m(q)}\right)<2 \in, \quad \forall q \geq q_{1} .
$$

As $\check{S}, \check{T}: \check{Z} \rightarrow P(\dot{Z})$ are semi- $\alpha_{*}$-dominated mappings on $\overline{B_{d_{l}}\left(c_{0}, \check{r}\right)}, \alpha_{*}\left(c_{\check{n}(q)}, \check{S} c_{\check{n}(q)}\right) \geq 1$ and $\alpha_{*}\left(c_{m(q)}, \check{T} c_{m(q)}\right) \geq 1$, for all $m, \check{n} \in N$. Now, by using Lemma 8 and condition (6), we get

$$
\begin{aligned}
& F(\epsilon) \leq F\left(H_{d_{l}}\left(\check{S} c_{\check{n}(q)}, \check{T} c_{m(q)}\right)\right) \leq \max \{\tau \\
& +\alpha_{*}\left(c_{\check{n}(q)}, \check{S} c_{\check{n}(q)}\right) F\left(H_{d_{l}}\left(\check{S} c_{\check{n}(q)}, \check{T} c_{m(q)}\right)\right), \tau \\
& \left.+\alpha_{*}\left(c_{m(q)}, \check{T} c_{m(q)}\right) F\left(H_{d_{l}}\left(\check{T} c_{m(q)}, \check{S} c_{\check{n}(q)}\right)\right)\right\}
\end{aligned}
$$




$$
\begin{aligned}
& \leq F\left[\eta_{1} d_{l}\left(c_{\check{n}(q)}, c_{m(q)}\right)+\eta_{2} d_{l}\left(c_{\check{n}(q)}, \check{S} c_{\check{n}(q)}\right)\right. \\
& +\eta_{3} d_{l}\left(c_{\check{n}(q)}, \check{T} c_{m(q)}\right) \\
& \left.+\eta_{4} \frac{d_{l}^{2}\left(c_{\check{n}(q)}, \check{S} c_{\check{n}(q)}\right) \cdot d_{l}\left(c_{m(q),} \check{T} c_{m(q)}\right)}{1+d_{l}^{2}\left(c_{\check{n}(q)}, c_{m(q)}\right)}\right]-\tau, \\
& F(\epsilon) \leq F\left[\eta_{1} d_{l}\left(c_{\check{n}(q)}, c_{m(q)}\right)+\eta_{2} d_{l}\left(c_{\check{n}(q)}, c_{\check{n}(q)+1}\right)\right. \\
& +\eta_{3} d_{l}\left(c_{\check{n}(q)}, c_{m(q)+1}\right) \\
& \left.+\eta_{4} \frac{d_{l}^{2}\left(c_{\check{n}(q)}, c_{\check{n}(q)+1}\right) \cdot d_{l}\left(c_{m(q)}, c_{m(q)+1}\right)}{1+d_{l}^{2}\left(c_{\check{n}(q)}, c_{m(q)}\right)}\right]-\tau, \\
& F(\epsilon) \leq F\left[\eta_{1} d_{l}\left(c_{\check{n}(q)}, c_{m(q)}\right)+\eta_{2} d_{l}\left(c_{\check{n}(q)}, c_{\check{n}(q)+1}\right)\right. \\
& +\eta_{3} d_{l}\left(c_{\check{n}(q)}, c_{m(q)}\right)+\eta_{3} d_{l}\left(c_{m(q)}, c_{m(q)+1}\right) \\
& \left.+\eta_{4} \frac{d_{l}^{2}\left(c_{\check{n}(q)}, c_{\check{n}(q)+1}\right) \cdot d_{l}\left(c_{m(q),} c_{m(q)+1}\right)}{1+d_{l}^{2}\left(c_{\check{n}(q)}, c_{m(q)}\right)}\right]-\tau,
\end{aligned}
$$

which implies that

$$
F(\epsilon) \leq F\left[2 \eta_{1} \in+\eta_{2} \in+3 \eta_{3} \in+\eta_{4} \in\right]-\tau .
$$

As $2 \eta_{1}+\eta_{2}+3 \eta_{3}+\eta_{4}<1$, we get

$$
2 \eta_{1} \in+\eta_{2} \in+3 \eta_{3} \in+\eta_{4} \in<\epsilon
$$

we deduce that

$$
F(\epsilon)<F(\epsilon)
$$

which is a contradiction. Thus, $\left\{\check{T} \check{S}\left(c_{\check{n}}\right)\right\}$ is a Cauchy sequence in $\left(\overline{B_{d_{l}}\left(c_{0}, \check{r}\right)}, d_{l}\right)$. Since $\left(\overline{B_{d_{l}}\left(c_{0}, \check{r}\right)}, d_{l}\right)$ is a complete metric space, so there exist $\bar{u} \in \overline{B_{d_{l}}\left(c_{0}, \check{r}\right)}$ such that $\left\{\check{T} \check{S}\left(c_{\check{n}}\right)\right\} \rightarrow \bar{u}$ as $\check{n} \rightarrow \infty$; then

$$
\lim _{\check{n} \rightarrow \infty} d_{l}\left(c_{\check{n}}, \bar{u}\right)=0
$$

Since $\alpha_{*}(\bar{u}, \check{T} \bar{u}) \geq 1$ and $\alpha_{*}\left(c_{2 \check{n}}, \check{S} c_{2 \check{n}}\right) \geq 1$, by using Lemma 8 and inequality (6), we have

$$
\begin{aligned}
& F\left(d_{l}\left(c_{2 \check{n}+1}, \check{T} \bar{u}\right)\right) \leq F\left(H_{d_{l}}\left(\check{S} c_{2 \check{n}}, \check{T} \bar{u}\right)\right) \leq \max \{\tau \\
& +\alpha_{*}\left(c_{2 \check{n}}, \check{S} c_{2 \check{n}}\right) F\left(H_{d_{l}}\left(\check{S} c_{2 \check{n}}, \check{T} \bar{u}\right)\right), \tau \\
& \left.+\alpha_{*}(\bar{u}, \check{T} \bar{u}) F\left(H_{d_{l}}\left(\check{T} \bar{u}, \check{S} c_{2 \check{n}}\right)\right)\right\} \\
& \leq F\left[\eta_{1} d_{l}\left(c_{2 \check{n}}, \bar{u}\right)+\eta_{2} d_{l}\left(c_{2 \check{n}}, \check{S} c_{2 \check{n}}\right)+\eta_{3} d_{l}\left(c_{2 \check{n}}, \check{T} \bar{u}\right)\right. \\
& \left.+\eta_{4} \frac{d_{l}^{2}\left(c_{2 \check{n}}, \check{S} c_{2 \check{n}}\right) \cdot d_{l}(\bar{u}, \check{T} \bar{u})}{1+d_{l}^{2}\left(c_{2 \check{n}}, \bar{u}\right)}\right]-\tau \\
& \leq F\left[\eta_{1} d_{l}\left(c_{2 \check{n}}, \bar{u}\right)+\eta_{2} d_{l}\left(c_{2 \check{n}}, \check{S} c_{2 \check{n}}\right)+\eta_{3} d_{l}\left(c_{2 \check{n}}, \bar{u}\right)\right. \\
& \left.+\eta_{3}(\bar{u}, \check{T} \bar{u})+\eta_{4} \frac{d_{l}^{2}\left(c_{2 \check{n}}, \check{S} c_{2 \check{n}}\right) \cdot d_{l}(\bar{u}, \check{T} \bar{u})}{1+d_{l}^{2}\left(c_{2 \check{n}}, \bar{u}\right)}\right]-\tau .
\end{aligned}
$$

By using (33), we get

$$
F\left(d_{l}(\bar{u}, \check{T} \bar{u})\right) \leq F\left[\eta_{3} d_{l}(\bar{u}, \check{T} \bar{u})\right]-\tau,
$$

which implies that

$$
d_{l}(\bar{u}, \check{T} \bar{u})<\eta_{3} d_{l}(\bar{u}, \check{T} \bar{u})<d_{l}(\bar{u}, \check{T} \bar{u}),
$$

which is a contradiction; hence $d_{l}(\bar{u}, \check{T} \bar{u})=0$ or $\bar{u} \epsilon$ $\check{T} \bar{u}$. Similarly, by using Lemma 8 , inequality (6), and the inequality

$$
d_{l}(\bar{u}, \check{S} \bar{u}) \leq d_{l}\left(\bar{u}, c_{2 \check{n}+2}\right)+d_{l}\left(c_{2 \check{n}+2}, \check{S} \bar{u}\right)
$$

we can show that $d_{l}(\bar{u}, \check{S} \bar{u})=0 . \bar{u} \in \check{S} \bar{u}$. Hence, $\check{S}$ and $\check{T}$ have a common fixed point $\bar{u}$ in $\overline{B_{d_{l}}\left(c_{0}, \check{r}\right)}$. Now,

$$
d_{l}(\bar{u}, \bar{u}) \leq d_{l}(\bar{u}, \check{T} \bar{u})+d_{l}(\check{T} \bar{u}, \bar{u}) \leq 0 .
$$

This implies that $d_{l}(\bar{u}, \bar{u})=0$.

Example 13. Let $\dot{Z}=Q^{+} \cup\{0\}$ and let $d_{l}: \dot{Z} \times \dot{Z} \rightarrow \dot{Z}^{\text {be the }}$ complete DMS on $Z$ defined by

$$
d_{l}(i, j)=i+j \quad \forall i, j \in \dot{Z} .
$$

Define the multivalued mapping, $\check{S}, \check{T}: \dot{Z} \times \dot{Z} \rightarrow P(\dot{Z})$, by

$$
\begin{aligned}
& \check{S} \dot{z}= \begin{cases}{\left[\frac{\dot{z}}{3}, \frac{2}{3} \dot{z}\right]} & \text { if } \dot{z} \in[0,7] \cap \dot{Z} \\
{[\dot{z}, \dot{z}+1]} & \text { if } \dot{z} \in(7, \infty) \cap \dot{Z},\end{cases} \\
& \check{T} \dot{z}= \begin{cases}{\left[\frac{\dot{z}}{4}, \frac{3}{4} \dot{z}\right]} & \text { if } \dot{z} \in[0,7] \cap \dot{Z} \\
{[\dot{z}+1, \dot{z}+3]} & \text { if } \dot{z} \in(7, \infty) \cap \dot{Z} .\end{cases}
\end{aligned}
$$


Considering that $x_{0}=1, \check{r}=8, \overline{B_{d_{l}}\left(x_{0}, \check{r}\right)}=[0,7] \cap \dot{Z}$. So we obtain a sequence $\left\{\check{T} \check{S}\left(x_{\check{n}}\right)\right\}=\{1,1 / 3,1 / 12,1 / 36, \ldots\}$ in $\dot{Z}$ generated by $x_{0}$. Also, $\overline{B_{d_{l}}\left(x_{0}, \check{r}\right)} \cap\left\{\check{T} \check{S}\left(x_{\check{n}}\right)\right\}=$ $\{1,1 / 3,1 / 12,1 / 36, \ldots\}$. Let

$$
\alpha(x, y)= \begin{cases}1 & \text { if } x, y \in[0,7] \cap \dot{Z} \\ \frac{3}{2} & \text { otherwise }\end{cases}
$$

$\tau \in(0,12 / 95], \eta_{1}=1 / 5, \eta_{2}=1 / 10, \eta_{3}=1 / 15, \eta_{4}=1 / 30$, and $\lambda=4 / 9$.

$$
d_{l}\left(x_{0}, \check{S} x_{0}\right)=\frac{4}{3}<\left(1-\frac{4}{9}\right) 8=(1-\lambda) \check{r} .
$$

Now, if $x, y \in \overline{B_{d_{l}}\left(x_{0}, \check{r}\right)} \cap\left\{\check{T} \check{S}\left(x_{\check{n}}\right)\right\}$, then we have the following cases.

Case 1. If $\max \left\{\tau+\alpha_{*}(x, \check{S} x) F\left(H_{d_{1}}(\check{S} x, \check{T} y)\right), \tau+\alpha_{*}(y\right.$, $\left.\check{T} y) F\left(H_{d_{l}}(\check{T} y, \check{S} x)\right)\right\}=\tau+\alpha_{*}(x, \check{S} x) F\left(H_{d_{l}}(\check{S} x, \check{T} y)\right)$, then we consider only

$$
\begin{aligned}
& H_{d_{l}}(\check{S} x, \check{T} y)=\max \left\{\sup _{a \in \check{S} x} d_{l}(a, \check{T} y), \underset{b \in \check{T} y}{\sup } d_{l}(\check{S} x, b)\right\} \\
& =\max \left\{\sup _{a \in \check{S} x} d_{l}\left(a,\left[\frac{y}{4}, \frac{3 y}{4}\right]\right),\right. \\
& \left.\quad \sup _{b \in \check{T} y} d_{l}\left(\left[\frac{x}{3}, \frac{2 x}{3}\right], b\right)\right\} \\
& =\max \left\{d_{l}\left(\frac{2 x}{3},\left[\frac{y}{4}, \frac{3 y}{4}\right]\right), d_{l}\left(\left[\frac{x}{3}, \frac{2 x}{3}\right], \frac{3 y}{4}\right)\right\} \\
& +\max \left\{d_{l}\left(\frac{2 x}{3}, \frac{y}{4}\right), d_{l}\left(\frac{x}{3}, \frac{3 y}{4}\right)\right\}=\max \left\{\frac{2 x}{3}\right. \\
& \left.+\frac{x}{3}+\frac{3 y}{4}\right\}<\frac{1}{5} d_{l}(x, y)+\frac{1}{10} d_{l}\left(x,\left[\frac{x}{3}, \frac{2}{3} x\right]\right) \\
& . \\
& \left.\left.\quad \frac{d_{l}^{2}(x,[x / 3,(2 / 3) x]) \cdot d_{l}(y,[y / 4,(3 / 4) y])}{1+d_{l}^{2}(x, y)}, \frac{3}{4} y\right]\right)+\frac{1}{30} \\
& =\frac{1}{5}(x+y)+\frac{2 x}{15}+\frac{4 x+y}{60}+\frac{5 x^{2} y^{2}}{54\left\{1+(x+y)^{2}\right\}}
\end{aligned}
$$

Thus,

$$
\begin{aligned}
H_{d_{l}}(\check{S} x, \check{T} y)< & \eta_{1} d_{l}(x, y)+\eta_{2} d_{l}(x, \check{S} x) \\
& +\eta_{3} d_{l}(x, \check{T} y) \\
& +\eta_{4} \frac{d_{l}^{2}(x, \check{S} x) \cdot d_{l}(y, \check{T} y)}{1+d_{l}^{2}(x, y)},
\end{aligned}
$$

which implies that

$$
\begin{gathered}
\tau+\ln \left(H_{d_{l}}(\check{S} x, \check{T} y)\right) \leq \ln \left(\eta_{1} d_{l}(x, y)\right. \\
+\eta_{2} d_{l}(x, \check{S} x)+\eta_{3} d_{l}(x, \check{T} y) \\
\left.+\eta_{4} \frac{d_{l}^{2}(x, \check{S} x) \cdot d_{l}(y, \check{T} y)}{1+d_{l}^{2}(x, y)}\right) .
\end{gathered}
$$

That is, if $F(\check{c})=\ln (\check{c})$, then

$$
\begin{aligned}
\tau+ & \alpha_{*}(x, \check{S} x) F\left(H_{d_{l}}(\check{S} x, \check{T} y)\right) \leq F\left(\eta_{1} d_{l}(x, y)\right. \\
& +\eta_{2} d_{l}(x, \check{S} x)+\eta_{3} d_{l}(x, \check{T} y) \\
& \left.+\eta_{4} \frac{d_{l}^{2}(x, \check{S} x) \cdot d_{l}(y, \check{T} y)}{1+d_{l}^{2}(x, y)}\right) .
\end{aligned}
$$

Case 2. If $\max \left\{\tau+\alpha_{*}(x, \check{S} x) F\left(H_{d_{l}}(\check{S} x, \check{T} y)\right), \tau+\alpha_{*}(y\right.$, $\left.\check{T} y) F\left(H_{d_{l}}(\check{T} y, \check{S} x)\right)\right\}=\tau+\alpha_{*}(y, \check{T} y) F\left(H_{d_{l}}(\check{T} y, \check{S} x)\right.$, then by using the similar arguments of Case 1 we can get the same results. Now, if $x=8, y=9 \in(7, \infty) \cap \dot{Z}$, then

$$
\begin{aligned}
\tau+ & \alpha_{*}(y, \check{T} y) F\left(H_{d_{l}}(\check{S} x, \check{T} y)\right)>F\left(\eta_{1} d_{l}(x, y)\right. \\
& +\eta_{2} d_{l}(x, \check{S} x)+\eta_{3} d_{l}(x, \check{T} y) \\
& \left.+\eta_{4} \frac{d_{l}^{2}(x, \check{S} x) \cdot d_{l}(y, \check{T} y)}{1+d_{l}^{2}(x, y)}\right) .
\end{aligned}
$$

And consequently condition (6) does not hold on Ź. Thus the mappings $\check{S}$ and $\check{T}$ satisfy all the conditions of Theorem 12 on closed ball rather than on whole space.

If we take $\check{S}=\check{T}$ in Theorem 12, then we are left with the result.

Corollary 14. Let $\left(Z, d_{l}\right)$ be a complete DMS. Suppose that there exists a function $\alpha: \dot{Z} \times \dot{Z} \rightarrow[0, \infty)$. Let $\check{r}>0$, let $c_{0} \in \overline{B_{d_{l}}\left(c_{0}, \check{r}\right)} \subseteq \dot{Z}$, and let $\check{S}: \dot{Z} \rightarrow P(\dot{Z})$ be the semi$\alpha_{*}$-dominated mappings on $\overline{B_{d_{1}}\left(c_{0}, \check{r}\right)}$. Assume that, for some $\tau>0$,

$$
\max \left\{\tau+\alpha_{*}(\breve{e}, \check{S} \breve{e}) F\left(H_{d_{l}}(\check{S} \breve{e}, \check{S} \hat{y})\right), \tau\right.
$$

$$
\begin{aligned}
& \left.+\alpha_{*}(\hat{y}, \check{S} \hat{y}) F\left(H_{d_{l}}(\check{S} \hat{y}, \check{S} \check{e})\right)\right\} \leq F\left(\eta_{1} d_{l}(\check{e}, \hat{y})\right. \\
& +\eta_{2} d_{l}(\check{e}, \check{S} \breve{e})+\eta_{3} d_{l}(\check{e}, \check{S} \breve{y}) \\
& \left.+\eta_{4} \frac{d_{l}^{2}(\check{e}, \check{S} \check{e}) \cdot d_{l}(\hat{y}, \check{S} \hat{y})}{1+d_{l}^{2}(x, \hat{y})}\right)
\end{aligned}
$$


for all $\breve{e}, \widehat{y} \in \overline{B_{d_{l}}\left(c_{0}, \check{r}\right)} \cap\left\{\check{S} \check{S}\left(c_{\check{n}}\right)\right\}$ with either $\alpha(\breve{e}, \widehat{y}) \geq 1$ or $\alpha(\hat{y}, \breve{e}) \geq 1$, where $\eta_{1}, \eta_{2}, \eta_{3}, \eta_{4}>0$ and $\eta_{1}+\eta_{2}+2 \eta_{3}+\eta_{4}<1$ and

$$
d_{l}\left(c_{0}, \check{S}_{c_{0}}\right) \leq(1-\lambda) \check{r}
$$

where $\lambda=\left(\left(\eta_{1}+\eta_{2}+\eta_{3}\right) /\left(1-\eta_{3}-\eta_{4}\right)\right)$ and $\eta_{3}+\eta_{4} \neq 1$. Then $\left\{\check{T} \check{S}\left(c_{\check{n}}\right)\right\}$ is a sequence in $\overline{B_{d_{l}}\left(c_{0}, \check{r}\right)}, \alpha\left(c_{\check{n}}, c_{\check{n}+1}\right) \geq 1$ for all $\check{n} \in N \cup\{0\}$, and $\left\{\check{S} \check{S}\left(c_{\check{n}}\right)\right\} \rightarrow \bar{u} \in \overline{B_{d_{l}}\left(c_{0}, \check{r}\right)}$. Also if inequality (48) holds for $\breve{e}$ and either $\alpha\left(c_{\check{n}}, \bar{u}\right) \geq 1$ or $\alpha\left(\bar{u}, c_{\check{n}}\right) \geq 1$ for all $\check{n} \in N \cup\{0\}$, then $\check{S}$ and $\check{T}$ have common fixed point $\bar{u}$ in

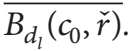
result.

If we take $\eta_{2}=0$ in Theorem 12, then we are left with the

Corollary 15. Let $\left(\dot{Z}, d_{l}\right)$ be a complete DMS. Suppose that there exists a function $\alpha: \dot{Z} \times \dot{Z} \rightarrow[0, \infty)$. Let $\check{r}>0$, let $c_{0} \in \overline{B_{d_{l}}\left(c_{0}, \check{r}\right)} \subseteq \dot{Z}$, and let $\check{S}, \check{T}: \dot{Z} \rightarrow P(\dot{Z})$ be the semi$\alpha_{*}$-dominated mappings on $\overline{B_{d_{l}}\left(c_{0}, \check{r}\right)}$. Assume that, for some $\tau>0$,

$$
\begin{aligned}
& \max \left\{\tau+\alpha_{*}(\breve{e}, \check{S} \breve{e}) F\left(H_{d_{l}}(\check{S} \breve{e}, \check{T} \hat{y})\right), \tau\right. \\
& \left.+\alpha_{*}(\widehat{y}, \check{T} \hat{y}) F\left(H_{d_{l}}(\check{T} \hat{y}, \check{S} \breve{e})\right)\right\} \leq F\left(\eta_{1} d_{l}(\breve{e}, \widehat{y})\right. \\
& \left.+\eta_{3} d_{l}(\breve{e}, \check{T} \hat{y})+\eta_{4} \frac{d_{l}^{2}(\breve{e}, \check{S} \breve{e}) \cdot d_{l}(\widehat{y}, \check{T} \hat{y})}{1+d_{l}^{2}(\breve{e}, \widehat{y})}\right)
\end{aligned}
$$

for all $\breve{e}, \widehat{y} \in \overline{B_{d_{l}}\left(c_{0}, \check{r}\right)} \cap\left\{\check{T} \check{S}\left(c_{\check{n}}\right)\right\}$ with either $\alpha(\breve{e}, \widehat{y}) \geq 1$ or $\alpha(\hat{y}, \breve{e}) \geq 1$, where $\eta_{1}, \eta_{3}, \eta_{4}>0$ and $\eta_{1}+2 \eta_{3}+\eta_{4}<1$ and

$$
d_{l}\left(c_{0}, \check{S} c_{0}\right) \leq(1-\lambda) \check{r}
$$

where $\lambda=\left(\left(\eta_{1}+\eta_{3}\right) /\left(1-\eta_{3}-\eta_{4}\right)\right)$ and $\eta_{3}+\eta_{4} \neq 1$. Then $\left\{\check{T} \check{S}\left(c_{\check{n}}\right)\right\}$ is a sequence in $\overline{B_{d_{l}}\left(c_{0}, \check{r}\right)}, \alpha\left(c_{\check{n}}, c_{\check{n}+1}\right) \geq 1$ for all $\check{n} \in N \cup\{0\}$, and $\left\{\check{T} \check{S}\left(c_{\check{n}}\right)\right\} \rightarrow \bar{u} \in \overline{B_{d_{1}}\left(c_{0}, \check{r}\right)}$. Also if inequality (50) holds for $\bar{u}$ and either $\alpha\left(c_{\check{n}}, \bar{u}\right) \geq 1$ or $\alpha\left(\bar{u}, c_{\check{n}}\right) \geq 1$ for all $\check{n} \in N \cup\{0\}$, then $\check{S}$ and $\check{T}$ have common fixed point $\bar{u}$ in $\overline{B_{d_{l}}\left(c_{0}, \check{r}\right)}$.

If we take $\eta_{3}=0$ in Theorem 12, then we are left with the result.

Corollary 16. Let $\left(\dot{Z}, d_{l}\right)$ be a complete DMS. Suppose that there exists a function $\alpha: \dot{Z} \times \dot{Z} \rightarrow[0, \infty)$. Let $\check{r}>0$, let $c_{0} \in \overline{B_{d_{l}}\left(c_{0}, \check{r}\right)} \subseteq \dot{Z}$, and let $\check{S}, \check{T}: \dot{Z} \rightarrow P(\dot{Z})$ be the semi$\alpha_{*}$-dominated mappings on $\overline{B_{d_{l}}\left(c_{0}, \check{r}\right)}$. Assume that, for some $\tau>0$,

$$
\begin{aligned}
& \max \left\{\tau+\alpha_{*}(\breve{e}, \check{S} \breve{e}) F\left(H_{d_{l}}(\check{S} \breve{e}, \check{T} \hat{y})\right), \tau\right. \\
& \left.+\alpha_{*}(\widehat{y}, \check{T} \hat{y}) F\left(H_{d_{l}}(\check{T} \hat{y}, \check{S} \breve{e})\right)\right\} \leq F\left(\eta_{1} d_{l}(\breve{e}, \widehat{y})\right. \\
& \left.+\eta_{2} d_{l}(\breve{e}, \check{S} \check{e})+\eta_{4} \frac{d_{l}^{2}(\breve{e}, \check{S} \check{e}) \cdot d_{l}(\widehat{y}, \check{T} \widehat{y})}{1+d_{l}^{2}(\breve{e}, \widehat{y})}\right)
\end{aligned}
$$

for all $\breve{e}, \widehat{y} \in \overline{B_{d_{l}}\left(c_{0}, \check{r}\right)} \cap\left\{\check{T} \check{S}\left(c_{\check{n}}\right)\right\}$ with either $\alpha(\breve{e}, \widehat{y}) \geq 1$ or $\alpha(\hat{y}, \breve{e}) \geq 1$, where $\eta_{1}, \eta_{2}, \eta_{4}>0$ and $\eta_{1}+\eta_{2}+\eta_{4}<1$ and

$$
d_{l}\left(c_{0}, \check{S} c_{0}\right) \leq(1-\lambda) \check{r}
$$

where $\lambda=\left(\left(\eta_{1}+\eta_{2}\right) /\left(1-\eta_{4}\right)\right)$ and $1-\eta_{4} \neq 0$. Then $\left\{\check{T} \check{S}\left(c_{\check{n}}\right)\right\}$ is a sequence in $\overline{B_{d_{l}}\left(c_{0}, \check{r}\right)}, \alpha\left(c_{\check{n}}, c_{\check{n}+1}\right) \geq 1$ for all $\check{n} \in N \cup\{0\}$, and $\left\{\check{T} \check{S}\left(c_{\check{n}}\right)\right\} \rightarrow \bar{u} \in \overline{B_{d_{l}}\left(c_{0}, \check{r}\right)}$. Also if inequality (52) holds for $\bar{u}$ and either $\alpha\left(c_{\check{n}}, \bar{u}\right) \geq 1$ or $\alpha\left(\bar{u}, c_{\check{n}}\right) \geq 1$ for all $\check{n} \in N \cup\{0\}$, then $\check{S}$ and $\check{T}$ have common fixed point $\bar{u}$ in $\overline{B_{d_{l}}\left(c_{0}, \check{r}\right)}$.

If we take $\eta_{4}=0$ in Theorem 12 , then we are left only with the result.

Corollary 17. Let $\left(\dot{Z}, d_{l}\right)$ be a complete DMS. Suppose that there exists a function $\alpha: \dot{Z} \times \dot{Z} \rightarrow[0, \infty)$. Let $\check{r}>0$, let $c_{0} \in \overline{B_{d_{l}}\left(c_{0}, \check{r}\right)} \subseteq \dot{Z}$, and let $\check{S}, \check{T}: \dot{Z} \rightarrow P(\dot{Z})$ be the semi$\alpha_{*}$-dominated mappings on $\overline{B_{d_{l}}\left(c_{0}, \check{r}\right)}$. Assume that, for some $\tau>0$,

$$
\begin{aligned}
& \max \left\{\tau+\alpha_{*}(\breve{e}, \check{S} \breve{e}) F\left(H_{d_{l}}(\check{S} \breve{e}, \check{T} \hat{y})\right), \tau\right. \\
& \left.\quad+\alpha_{*}(\widehat{y}, \check{T} \hat{y}) F\left(H_{d_{l}}(\check{T} \widehat{y}, \check{S} \breve{e})\right)\right\} \leq F\left(\eta_{1} d_{l}(\breve{e}, \widehat{y})\right. \\
& \left.+\eta_{2} d_{l}(\breve{e}, \check{S} \breve{e})+\eta_{3} d_{l}(\breve{e}, \check{T} \widehat{y})\right)
\end{aligned}
$$

for all $\breve{e}, \widehat{y} \in \overline{B_{d_{l}}\left(c_{0}, \check{r}\right)} \cap\left\{\check{T} \check{S}\left(c_{\check{n}}\right)\right\}$ with either $\alpha(\breve{e}, \widehat{y}) \geq 1$ or $\alpha(\widehat{y}, \breve{e}) \geq 1$, where $\eta_{1}, \eta_{2}, \eta_{3}>0$ and $\eta_{1}+\eta_{2}+2 \eta_{3}<1$ and

$$
d_{l}\left(c_{0}, \check{S} c_{0}\right) \leq(1-\lambda) \check{r}
$$

where $\lambda=\left(\left(\eta_{1}+\eta_{2}+\eta_{3}\right) /\left(1-\eta_{3}\right)\right)$ and $1-\eta_{3} \neq 0$. Then $\left\{\check{T} \check{S}\left(c_{\check{n}}\right)\right\}$ is a sequence in $\overline{B_{d_{l}}\left(c_{0}, \check{r}\right)}, \alpha\left(c_{\check{n}}, c_{\check{n}+1}\right) \geq 1$ for all $\check{n} \in N \cup\{0\}$, and $\left\{\check{T} \check{S}\left(c_{\check{n}}\right)\right\} \rightarrow \bar{u} \in \overline{B_{d_{l}}\left(\bar{u}_{0}, \check{r}\right)}$. Also if inequality (54) holds for $\bar{u}$ and either $\alpha\left(c_{\check{n}}, \bar{u}\right) \geq 1$ or $\alpha\left(\bar{u}, c_{\check{n}}\right) \geq 1$ for all $\check{n} \in N \cup\{0\}$, then $\check{S}$ and $\check{T}$ have common fixed point $\bar{u}$ in $\overline{B_{d_{l}}\left(c_{0}, \check{r}\right)}$.

\section{Fixed Point Results for Graphic Contractions}

In this section, we present an application of Theorem 12 in graph theory. Jachymski [21], proved the result concerning for contraction mappings on metric space with a graph. Hussain et al. [16] introduced the fixed points theorem for $\psi_{b}$-graphic contraction and gave an application to system of integral equations. A graph $\breve{G}$ is a connected graph if there must exist a path among any two different vertices (for details, see $[13,32])$.

Definition 18. Let $\dot{Z}$ be a nonempty set and let $\breve{G}=$ $(V(\breve{G}), E(\breve{G}))$ be a graph such that $V(\breve{G})=\dot{Z}$, and $\check{S}: \dot{Z} \rightarrow$ $P\left(Z^{\prime}\right)$ is said to be multigraph-dominated if $(\dot{z}, \grave{u}) \in E(\breve{G})$, for all $\grave{u} \in \check{S} \check{z}$. 
Theorem 19. Let $\left(\dot{Z}, d_{l}\right)$ be a complete DMS endowed with a graph $\breve{G}$. Suppose that there exists a function $\alpha: \dot{Z} \times \dot{Z} \rightarrow$ $[0, \infty)$. Let $\check{r}>0$, let $c_{0} \in \overline{B_{d_{1}}\left(c_{0}, \check{r}\right)}, \check{S}, \check{T}: \check{Z} \rightarrow P(\dot{Z})$, and assume a sequence $\left\{\check{T} \check{S}\left(c_{\check{n}}\right)\right\}$ in $Z$ generated by $c_{0}$, with $\left(c_{0}, c_{1}\right) \in$ $E(\breve{G})$. Assume that the following hold:

(i) $\check{S}$ and $\check{T}$ are multigraph-dominated for all $\breve{e}, \hat{y} \in$ $\overline{B_{d_{1}}\left(c_{0}, \check{r}\right)} \cap\left\{\check{T} \check{S}\left(c_{\check{n}}\right)\right\}$.

(ii) There exist some $\eta_{1}, \eta_{2}, \eta_{3}, \eta_{4}, \tau>0$, and

$$
\begin{aligned}
& \max \left\{\tau+\alpha_{*}(\breve{e}, \check{S} \breve{e}) F\left(H_{d_{l}}(\check{S} \check{e}, \check{T} \hat{y})\right), \tau\right. \\
& \left.+\alpha_{*}(\widehat{y}, \check{T} \hat{y}) F\left(H_{d_{l}}(\check{T} \hat{y}, \check{S} \check{e})\right)\right\} \leq F\left(\eta_{1} d_{l}(\breve{e}, \widehat{y})\right. \\
& +\eta_{2} d_{l}(\breve{e}, \check{S} \breve{e})+\eta_{3} d_{l}(\breve{e}, \check{T} \hat{y}) \\
& \left.+\eta_{4} \frac{d_{l}^{2}(\breve{e}, \check{S} \breve{e}) \cdot d_{l}(\widehat{y}, \check{T} \hat{y})}{1+d_{l}^{2}(\breve{e}, \hat{y})}\right)
\end{aligned}
$$

for all $\breve{e}, \hat{y} \in \overline{B_{d_{l}}\left(c_{0}, \check{r}\right)} \cap\left\{\check{T} \check{S}\left(c_{\breve{n}}\right)\right\}$ and $(\breve{e}, \widehat{y}) \in E(\breve{G})$ or $(\widehat{y}, \breve{e}) \in$ $E(\breve{G})$.

(iii) $\sum_{i=0}^{\check{n}} \lambda^{i}\left(d_{l}\left(c_{0}, \check{S} c_{0}\right)\right) \leq \check{r}$ for all $\check{n} \in N \cup\{0\}$.

Then, $\left\{\check{T} \check{S}\left(c_{\check{n}}\right)\right\}$ is a sequence in $\overline{B_{d_{l}}\left(c_{0}, \check{r}\right)},\left(c_{\check{n}}, c_{\check{n}+1}\right) \in E(\breve{G})$, and $\left\{\check{T} \check{S}\left(c_{\check{n}}\right)\right\} \rightarrow m^{*}$. Also, if inequality (56) holds for $m^{*}$ and $\left(c_{\breve{n}}, m^{*}\right) \in E(\breve{G})$ or $\left(m^{*}, c_{\check{n}}\right) \in E(\breve{G})$ for all $\check{n} \in N \cup\{0\}$, then $\check{S}$ and $\check{T}$ have common fixed point $m^{*}$ in $\overline{B_{d_{l}}\left(c_{0}, \check{r}\right)}$ and $d_{l}\left(m^{*}, m^{*}\right)=0$.

Proof. Define $\alpha: \dot{Z} \times \dot{Z} \rightarrow[0, \infty)$ by

$$
\begin{aligned}
& \alpha(\breve{e}, \hat{y}) \\
& = \begin{cases}1, & \text { if } \breve{e} \in \overline{B_{d_{l}}\left(c_{0}, \breve{r}\right)},(\breve{e}, \widehat{y}) \in E(\breve{G}) \text { or }(\widehat{y}, \breve{e}) \in E(\breve{G}) \\
0, & \text { otherwise. }\end{cases}
\end{aligned}
$$

As $\check{S}$ and $\check{T}$ are semigraph-dominated on $\overline{B_{d_{l}}\left(c_{0}, \check{r}\right)}$, then, for $\breve{e} \in \overline{B_{d_{l}}\left(c_{0}, \check{r}\right)},(\breve{e}, \hat{y}) \in E(\breve{G})$ for all $\hat{y} \in \check{S} \breve{e}$ and $(\breve{e}, \hat{y}) \in E(\breve{G})$ for all $\hat{y} \in \check{T} \breve{e}$. So, $\alpha(\breve{e}, \widehat{y})=1$ for all $\hat{y} \in \check{S} \breve{e}$ and $\alpha(\breve{e}, \widehat{y})=1$ for all $\hat{y} \in \check{T} \breve{e}$. This implies that $\inf \{\alpha(\breve{e}, \hat{y}): \hat{y} \in \check{S} \breve{e}\}=1$ and $\inf \{\alpha(\breve{e}, \hat{y}): \hat{y} \in \check{T} \breve{e}\}=1$. Hence $\alpha_{*}(\breve{e}, \breve{S} \breve{e})=1, \alpha_{*}(\breve{e}, \breve{T} \breve{e})=1$ for all $\breve{e} \in \overline{B_{d_{l}}\left(c_{0}, \check{r}\right)}$. So, $\check{S}, \check{T}: \check{Z} \rightarrow P(\check{Z})$ are the semi- $\alpha_{*}$ dominated mappings on $\overline{B_{d_{l}}\left(c_{0}, \check{r}\right)}$. Moreover, inequality (56) can be written as

$$
\begin{aligned}
& \max \left\{\tau+\alpha_{*}(\breve{e}, \check{S} \check{e}) F\left(H_{d_{l}}(\check{S} \breve{e}, \check{T} \hat{y})\right), \tau\right. \\
& \left.+\alpha_{*}(\widehat{y}, \check{T} \hat{y}) F\left(H_{d_{l}}(\check{T} \hat{y}, \check{S} \breve{e})\right)\right\} \leq F\left(\eta_{1} d_{l}(\breve{e}, \widehat{y})\right. \\
& +\eta_{2} d_{l}(\breve{e}, \check{S} \breve{e})+\eta_{3} d_{l}(\breve{e}, \check{T} \hat{y}) \\
& \left.+\eta_{4} \frac{d_{l}^{2}(\breve{e}, \check{S} \breve{e}) \cdot d_{l}(\hat{y}, \check{T} \hat{y})}{1+d_{l}^{2}(\breve{e}, \widehat{y})}\right)
\end{aligned}
$$

for all elements $\breve{e}, \hat{y}$ in $\overline{B_{d_{l}}\left(c_{0}, \check{r}\right)} \cap\left\{\check{T} \check{S}\left(x_{\breve{n}}\right)\right\}$ with either $\alpha(\breve{e}, \hat{y}) \geq 1$ or $\alpha(\widehat{y}, \breve{e}) \geq 1$. Also, (iii) holds. Then, by Theorem 12, we have that $\left\{\check{T} \check{S}\left(c_{\check{n}}\right)\right\}$ is a sequence in $\overline{B_{d_{l}}\left(c_{0}, \check{r}\right)}$ and $\left\{\check{T} \check{S}\left(c_{\check{n}}\right)\right\} \rightarrow m^{*} \in \overline{B_{d_{l}}\left(c_{0}, \check{r}\right)}$. Now, $c_{\check{n}}, m^{*} \in \overline{B_{d_{l}}\left(c_{0}, \check{r}\right)}$ and either $\left(c_{\breve{n}}, m^{*}\right) \in E(\breve{G})$ or $\left(m^{*}, c_{\breve{n}}\right) \in E(\breve{G})$ implies that either $\alpha\left(c_{\breve{n}}, m^{*}\right) \geq 1$ or $\alpha\left(m^{*}, c_{\check{n}}\right) \geq 1$. So, all the conditions of Theorem 12 are satisfied. Hence, by Theorem 12, $\check{S}$ and $\check{T}$ have a common fixed point $m^{*}$ in $\overline{B_{d_{l}}\left(c_{0}, \check{r}\right)}$ and $d_{l}\left(m^{*}, m^{*}\right)=$ 0 .

\section{Fixed Point Results for Single Valued Mapping}

In this section, we discuss some new fixed point results for single valued mapping in complete DMS.

Theorem 20. Let $\left(Z, d_{l}\right)$ be a complete DMS. Suppose that there exists a function $\alpha: \dot{Z} \times \dot{Z} \rightarrow[0, \infty)$. Let $\check{r}>0$, let $c_{0} \in \overline{B_{d_{1}}\left(c_{0}, \check{r}\right)} \subseteq \dot{Z}$, and let $\check{S}, \check{T}: \dot{Z} \rightarrow \dot{Z}$ be the semi$\alpha_{*}$-dominated mappings on $\overline{B_{d_{l}}\left(c_{0}, \check{r}\right)}$. Assume that, for some $\tau>0$,

$$
\begin{aligned}
& \max \left\{\tau+\alpha_{*}(\check{e}, \check{S} \check{e}) F\left(d_{l}(\check{S} \check{e}, \check{T} \hat{y})\right), \tau\right. \\
& \left.+\alpha_{*}(\widehat{y}, \check{T} \widehat{y}) F\left(d_{l}(\check{T} \hat{y}, \check{S} \breve{e})\right)\right\} \leq F\left(\eta_{1} d_{l}(\breve{e}, \widehat{y})\right. \\
& +\eta_{2} d_{l}(\breve{e}, \check{S} \breve{e})+\eta_{3} d_{l}(\breve{e}, \check{T} \hat{y}) \\
& \left.+\eta_{4} \frac{d_{l}^{2}(\breve{e}, \check{S} \check{e}) \cdot d_{l}(\hat{y}, \check{T} \hat{y})}{1+d_{l}^{2}(\breve{e}, \widehat{y})}\right)
\end{aligned}
$$

for all $\breve{e}, \widehat{y} \in \overline{B_{d_{l}}\left(c_{0}, \breve{r}\right)} \cap\left\{c_{\breve{n}}\right\}$ with either $\alpha(\breve{e}, \widehat{y}) \geq 1$ or $\alpha(\hat{y}, \breve{e}) \geq$ 1 , where $\eta_{1}, \eta_{2}, \eta_{3}, \eta_{4}>0$ and $\eta_{1}+\eta_{2}+2 \eta_{3}+\eta_{4}<1$ and

$$
d_{l}\left(c_{0}, \check{S} c_{0}\right) \leq(1-\lambda) \check{r}
$$

where $\lambda=\left(\left(\eta_{1}+\eta_{2}+\eta_{3}\right) /\left(1-\eta_{3}-\eta_{4}\right)\right)$ and $\eta_{3}+\eta_{4} \neq 1$. Then $\left\{c_{\check{n}}\right\}$ is a sequence in $\overline{B_{d_{l}}\left(c_{0}, \check{r}\right)}, \alpha\left(c_{\check{n}}, c_{\check{n}+1}\right) \geq 1$ for all $\check{n} \in N \cup\{0\}$, and $\left\{c_{\breve{n}}\right\} \rightarrow v \in \overline{B_{d_{l}}\left(c_{0}, \check{r}\right)}$. Also if inequality (59) holds for $v$ and either $\alpha\left(c_{\tilde{n}}, v\right) \geq 1$ or $\alpha\left(v, c_{\tilde{n}}\right) \geq 1$ for all $\check{n} \in N \cup\{0\}$, then $\check{S}$ and $\check{T}$ have common fixed point $v$ in $\overline{B_{d_{l}}\left(c_{0}, \check{r}\right)}$.

Proof. The proof of the above Theorem is the same as Theorem 12.

If we take $\check{S}=\check{T}$ in Theorem 20 , then we have the following result.

Corollary 21. Let $\left(Z, d_{l}\right)$ be a complete DMS. Suppose that there exists a function $\alpha: \dot{Z} \times \dot{Z} \rightarrow[0, \infty)$. Let $\check{r}>0$, let 
$c_{0} \in \overline{\mathrm{B}_{d_{l}}\left(c_{0}, \check{r}\right)} \subseteq \dot{Z}$, and let $\check{S}: \dot{Z} \rightarrow \dot{Z}$ be the semi- $\alpha_{*}-$ dominated mappings on $\overline{B_{d_{l}}\left(c_{0}, \check{r}\right)}$. Assume that, for some $\tau>0$,

$$
\begin{aligned}
& \max \left\{\tau+\alpha_{*}(\breve{e}, \check{S} \breve{e}) F\left(d_{l}(\check{S} \breve{e}, \check{S} \widehat{y})\right), \tau\right. \\
& \left.+\alpha_{*}(\widehat{y}, \check{S} \hat{y}) F\left(d_{l}(\check{S} \hat{y}, \check{S} \check{e})\right)\right\} \leq F\left(\eta_{1} d_{l}(\breve{e}, \hat{y})\right. \\
& +\eta_{2} d_{l}(\breve{e}, \check{S} \breve{e})+\eta_{3} d_{l}(\breve{e}, \check{S} \hat{y}) \\
& \left.+\eta_{4} \frac{d_{l}^{2}(\breve{e}, \check{S} \breve{e}) \cdot d_{l}(\hat{y}, \check{S} \widehat{y})}{1+d_{l}^{2}(\breve{e}, \hat{y})}\right)
\end{aligned}
$$

for all $\breve{e}, \hat{y} \in \overline{B_{d_{l}}\left(c_{0}, \check{r}\right)} \cap\left\{c_{\breve{n}}\right\}$ with either $\alpha(\breve{e}, \widehat{y}) \geq 1$ or $\alpha(\hat{y}, \breve{e}) \geq 1$, where $\eta_{1}, \eta_{2}, \eta_{3}, \eta_{4}>0$ and $\eta_{1}+\eta_{2}+\eta_{3}+\eta_{4}<1$ and

$$
d_{l}\left(c_{0}, \check{S} c_{0}\right) \leq(1-\lambda) \check{r}
$$

where $\lambda=\left(\left(\eta_{1}+\eta_{2}\right) /\left(1-\eta_{3}-\eta_{4}\right)\right)$ and $\eta_{3}+\eta_{4} \neq 1$. Then $\left\{c_{\grave{n}}\right\}$ is a sequence in $\overline{B_{d_{1}}\left(c_{0}, \check{r}\right)}, \alpha\left(c_{\check{n}}, c_{\check{n}+1}\right) \geq 1$ for all $\check{n} \in N \cup\{0\}$, and $\left\{c_{\tilde{n}}\right\} \rightarrow v \in \overline{B_{d_{1}}\left(c_{0}, \check{r}\right)}$. Also if inequality (61) holds for $v$ and either $\alpha\left(c_{\check{n}}, v\right) \geq 1$ or $\alpha\left(v, c_{\check{n}}\right) \geq 1$ for all $\check{n} \in N \cup\{0\}$, then $\check{S}$ and $\check{T}$ have common fixed point $v$ in $\overline{B_{d_{l}}\left(c_{0}, \check{r}\right) \text {. }}$ result.

If we take $\eta_{2}=0$ in Theorem 20, then we are left with the

Corollary 22. Let $\left(\dot{Z}, d_{l}\right)$ be a complete DMS. Suppose that there exists a function $\alpha: \dot{Z} \times \dot{Z} \rightarrow[0, \infty)$. Let $\check{r}>0$, let $c_{0} \in \overline{B_{d_{l}}\left(c_{0}, \check{r}\right)} \subseteq \dot{Z}$, and let $\check{S}, \check{T}: \dot{Z} \rightarrow \dot{Z}$ be the semi$\alpha_{*}$-dominated mappings on $\overline{B_{d_{l}}\left(c_{0}, \check{r}\right)}$. Assume that, for some $\tau>0$,

$$
\begin{aligned}
& \max \left\{\tau+\alpha_{*}(\breve{e}, \check{S} \breve{e}) F\left(d_{l}(\check{S} \breve{e}, \check{T} \widehat{y})\right), \tau\right. \\
& \left.+\alpha_{*}(\widehat{y}, \check{T} \hat{y}) F\left(d_{l}(\check{T} \hat{y}, \check{S} \breve{e})\right)\right\} \leq F\left(\eta_{1} d_{l}(\breve{e}, \widehat{y})\right. \\
& \left.+\eta_{3} d_{l}(\check{e}, \check{T} \hat{y})+\eta_{4} \frac{d_{l}^{2}(\breve{e}, \check{S} \breve{e}) \cdot d_{l}(\widehat{y}, \check{T} \hat{y})}{1+d_{l}^{2}(\breve{e}, \hat{y})}\right)
\end{aligned}
$$

for all $\breve{e}, \widehat{y} \in \overline{B_{d_{1}}\left(c_{0}, \check{r}\right)} \cap\left\{c_{\check{n}}\right\}$ with either $\alpha(\breve{e}, \widehat{y}) \geq 1$ or $\alpha(\widehat{y}, \breve{e}) \geq$ 1 , where $\eta_{1}, \eta_{3}, \eta_{4}>0$ and $\eta_{1}+2 \eta_{3}+\eta_{4}<1$ and

$$
d_{l}\left(c_{0}, \check{S} c_{0}\right) \leq(1-\lambda) \check{r}
$$

where $\lambda=\left(\left(\eta_{1}+\eta_{3}\right) /\left(1-\eta_{3}-\eta_{4}\right)\right)$ and $\eta_{3}+\eta_{4} \neq 1$. Then $\left\{c_{\check{n}}\right\}$ is a sequence in $\overline{B_{d_{l}}\left(c_{0}, \check{r}\right)}, \alpha\left(c_{\check{n}}, c_{\check{n}+1}\right) \geq 1$ for all $\check{n} \in N \cup\{0\}$, and $\left\{c_{\check{n}}\right\} \rightarrow v \in \overline{B_{d_{l}}\left(c_{0}, \check{r}\right)}$. Also if inequality (63) holds for $v$ and either $\alpha\left(c_{\check{n}}, v\right) \geq 1$ or $\alpha\left(v, c_{\check{n}}\right) \geq 1$ for all $\check{n} \in N \cup\{0\}$, then $\check{S}$ and $\check{T}$ have common fixed point $v$ in $\overline{B_{d_{l}}\left(c_{0}, \check{r}\right)}$. result.

If we take $\eta_{3}=0$ in Theorem 20 , then we are left with the
Corollary 23. Let $\left(\dot{Z}, d_{l}\right)$ be a complete DMS. Suppose that there exists a function $\alpha: \dot{Z} \times \dot{Z} \rightarrow[0, \infty)$. Let $\check{r}>0$, let $c_{0} \in \overline{B_{d_{1}}\left(c_{0}, \check{r}\right)} \subseteq \dot{Z}$, and let $\check{S}, \check{T}: \dot{Z} \rightarrow \dot{Z}$ be the semi$\alpha_{*}$-dominated mappings on $\overline{B_{d_{l}}\left(c_{0}, \check{r}\right)}$. Assume that, for some $\tau>0$,

$$
\begin{aligned}
& \max \left\{\tau+\alpha_{*}(\breve{e}, \check{S} \breve{e}) F\left(d_{l}(\check{S} \breve{e}, \check{T} \hat{y})\right), \tau\right. \\
& \left.+\alpha_{*}(\widehat{y}, \check{T} \hat{y}) F\left(d_{l}(\check{T} \hat{y}, \check{S} \check{e})\right)\right\} \leq F\left(\eta_{1} d_{l}(\breve{e}, \widehat{y})\right. \\
& \left.+\eta_{2} d_{l}(\check{e}, \check{S} \breve{e})+\eta_{4} \frac{d_{l}^{2}(\breve{e}, \check{S} \breve{e}) \cdot d_{l}(\hat{y}, \check{T} \hat{y})}{1+d_{l}^{2}(\breve{e}, \widehat{y})}\right)
\end{aligned}
$$

for all $\breve{e}, \hat{y} \in \overline{B_{\mathrm{d}_{l}}\left(c_{0}, \check{r}\right)} \cap\left\{c_{\check{n}}\right\}$ with either $\alpha(\breve{e}, \widehat{y}) \geq 1$ or $\alpha(\widehat{y}, \breve{e}) \geq$ 1 , where $\eta_{1}, \eta_{2}, \eta_{4}>0$ and $\eta_{1}+\eta_{2}+\eta_{4}<1$ and

$$
d_{l}\left(c_{0}, \check{S} c_{0}\right) \leq(1-\lambda) \check{r}
$$

where $\lambda=\left(\left(\eta_{1}+\eta_{2}\right) /\left(1-\eta_{4}\right)\right)$ and $1-\eta_{4} \neq 0$. Then $\left\{c_{\grave{n}}\right\}$ is a sequence in $\overline{B_{d_{l}}\left(c_{0}, \check{r}\right)}, \alpha\left(c_{\check{n}}, c_{\check{n}+1}\right) \geq 1$ for all $\check{n} \in N \cup\{0\}$, and $\left\{c_{\breve{n}}\right\} \rightarrow v \in \overline{B_{d_{1}}\left(c_{0}, \check{r}\right)}$. Also if inequality (65) holds for $v$ and either $\alpha\left(c_{\check{n}}, v\right) \geq 1$ or $\alpha\left(v, c_{\check{n}}\right) \geq 1$ for all $\check{n} \in N \cup\{0\}$, then $\check{S}$ and $\check{T}$ have common fixed point $v$ in $\overline{B_{d_{l}}\left(c_{0}, \check{r}\right)}$. result.

If we take $\eta_{4}=0$ in Theorem 20, then we are left with the

Corollary 24. Let $\left(Z, d_{l}\right)$ be a complete DMS. Suppose that there exists a function $\alpha: \dot{Z} \times \dot{Z} \rightarrow[0, \infty)$. Let $\check{r}>0$, let $c_{0} \in$ $\overline{B_{d_{l}}\left(c_{0}, \check{r}\right)} \subseteq \dot{Z}$, and let $\check{S}, \check{T}: \dot{Z} \rightarrow \dot{Z}$ be the semi- $\alpha_{*}$-dominated mappings on $\overline{B_{d_{l}}\left(c_{0}, \check{r}\right)}$. Assume that, for some $\tau>0$,

$$
\begin{aligned}
\max & \left\{\tau+\alpha_{*}(\check{e}, \check{S} \breve{e}) F\left(d_{l}(\check{S} \breve{e}, \check{T} \hat{y})\right), \tau\right. \\
& \left.+\alpha_{*}(\widehat{y}, \check{T} \hat{y}) F\left(d_{l}(\check{T} \hat{y}, \check{S} \check{e})\right)\right\} \leq F\left(\eta_{1} d_{l}(\breve{e}, \hat{y})\right. \\
& \left.+\eta_{2} d_{l}(\breve{e}, \check{S} \check{e})+\eta_{3} d_{l}(\breve{e}, \check{T} \hat{y})\right)
\end{aligned}
$$

for all $\breve{e}, \widehat{y} \in \overline{B_{d_{l}}\left(\breve{e}_{0}, \check{r}\right)} \cap\left\{c_{\breve{n}}\right\}$ with either $\alpha(\breve{e}, \widehat{y}) \geq 1$ or $\alpha(\widehat{y}, \breve{e}) \geq$ 1 , where $\eta_{1}, \eta_{2}, \eta_{3}>0$ and $\eta_{1}+\eta_{2}+2 \eta_{3}<1$ and

$$
d_{l}\left(c_{0}, \check{S} c_{0}\right) \leq(1-\lambda) \check{r}
$$

where $\lambda=\left(\left(\eta_{1}+\eta_{2}+\eta_{3}\right) /\left(1-\eta_{3}\right)\right)$ and $1-\eta_{3} \neq 0$. Then $\left\{c_{\grave{n}}\right\}$ is a sequence in $\overline{B_{d_{1}}\left(c_{0}, \check{r}\right)}, \alpha\left(c_{\check{n}}, c_{\check{n}+1}\right) \geq 1$ for all $\check{n} \in N \cup\{0\}$, and $\left\{c_{\breve{n}}\right\} \rightarrow v \in \overline{B_{d_{1}}\left(c_{0}, \check{r}\right)}$. Also if inequality (67) holds for $v$ and either $\alpha\left(c_{\check{n}}, v\right) \geq 1$ or $\alpha\left(v, c_{\check{n}}\right) \geq 1$ for all $\check{n} \in N \cup\{0\}$, then $\check{S}$ and $\check{T}$ have common fixed point $v$ in $\overline{B_{d_{l}}\left(c_{0}, \check{r}\right)}$. 


\section{Application to the Systems of Integral Equations}

Theorem 25. Let $\left(\dot{Z}, d_{l}\right)$ be a complete DMS. Let $\check{r}>0$, let $c_{0} \in \overline{B_{d_{l}}\left(c_{0}, \check{r}\right)} \subseteq \dot{Z}$, and let $\check{S}, \check{T}: \dot{Z} \rightarrow \dot{Z}$ be the dominated mappings on $\overline{B_{d_{l}}\left(c_{0}, \check{r}\right)}$. Assume that, for some $\tau>0$,

$$
\begin{aligned}
\max & \left\{\tau+F\left(d_{l}(\check{S} \breve{e}, \check{T} \hat{y})\right), \tau+F\left(d_{l}(\check{T} \hat{y}, \check{S} \breve{e})\right)\right\} \\
& \leq F\left(\eta_{1} d_{l}(\check{e}, \widehat{y})+\eta_{2} d_{l}(\breve{e}, \check{S} \breve{e})+\eta_{3} d_{l}(\check{e}, \check{T} \hat{y})\right. \\
& \left.+\eta_{4} \frac{d_{l}^{2}(\check{e}, \check{S} \breve{e}) \cdot d_{l}(\widehat{y}, \check{T} \hat{y})}{1+d_{l}^{2}(\breve{e}, \widehat{y})}\right)
\end{aligned}
$$

for all $\breve{e}, \hat{y} \in \overline{B_{d_{1}}\left(c_{0}, \check{r}\right)} \cap\left\{c_{\breve{n}}\right\}$ with $\eta_{1}, \eta_{2}, \eta_{3}, \eta_{4}>0$, where $\eta_{1}+$ $\eta_{2}+2 \eta_{3}+\eta_{4}<1$ and

$$
d_{l}\left(c_{0}, \check{S} c_{0}\right) \leq(1-\lambda) \check{r}
$$

where $\lambda=\left(\left(\eta_{1}+\eta_{2}+\eta_{3}\right) /\left(1-\eta_{3}-\eta_{4}\right)\right)$ and $\eta_{3}+\eta_{4} \neq 1$. Then $\left\{c_{\check{n}}\right\}$ is a sequence in $\overline{B_{d_{l}}\left(c_{0}, \check{r}\right)}$, for all $\check{n} \in N \cup\{0\}$ and $\left\{c_{\check{n}}\right\} \rightarrow v \in \overline{B_{d_{l}}\left(c_{0}, \check{r}\right)}$. Then $\check{S}$ and $\check{T}$ have common fixed point $v$ in $\overline{B_{d_{l}}\left(c_{0}, \check{r}\right)}$.

Proof. The proof of the above theorem is the same as the theorem proven in the previous section. In this section, we discuss the application of fixed point Theorem 25 in form of Volterra type integral equations.

$$
\begin{aligned}
& \breve{u}(k)=\int_{0}^{k} H_{1}(k, h, \breve{u}(h)) d h, \\
& \check{c}(k)=\int_{0}^{k} H_{2}(k, h, \check{c}(h)) d h
\end{aligned}
$$

for all $k \in[0,1]$. We find the solution of (71). Let $\dot{Z}=\dot{C}\left([0,1], \mathbb{R}_{+}\right)$be the set of all continuous functions on $[0,1]$, endowed with the complete dislocated metric. For $\breve{u} \in \dot{C}\left([0,1], \mathbb{R}_{+}\right)$, define supremum norm as $\|\breve{u}\|_{\tau}=$ $\sup _{k \in[0,1]}\left\{|\breve{u}(k)| e^{-\tau k}\right\}$, where $\tau>0$ is taken arbitrarily. Then define

$$
d_{\tau}(\breve{u}, \check{c})=\sup _{k \in[0,1]}\left\{|\breve{u}(k)+\check{c}(k)| e^{-\tau k}\right\}=\|\breve{u}+\check{c}\|_{\tau}
$$

for all $\breve{u}, \check{c} \in \dot{C}\left([0,1], \mathbb{R}_{+}\right)$, and, with these settings, $\left(C\left([0,1], \mathbb{R}_{+}\right), d_{\tau}\right)$ becomes a complete DMS.

Now we prove the following theorem to ensure the existence of solution of integral equations.

Theorem 26. Assume that the following conditions are satisfied:

(i) $H_{1}, H_{2}:[0,1] \times[0,1] \times \dot{C}\left([0,1], \mathbb{R}_{+}\right) \rightarrow \mathbb{R}$. (ii) Define

$$
\begin{aligned}
& \check{S} \breve{u}(k)=\int_{0}^{k} H_{1}(k, h, \breve{u}(h)) d h, \\
& \check{T} \check{c}(k)=\int_{0}^{k} H_{2}(k, h, \check{c}(h)) d h .
\end{aligned}
$$

Suppose that there exists $\tau>0$, such that

$$
\left|H_{1}(k, h, \breve{u})+H_{2}(k, h, \check{c})\right| \leq \frac{\tau N(\breve{u}, \check{c})}{\left(\tau \sqrt{\|N(\breve{u}, \check{c})\|_{\tau}}+1\right)^{2}}
$$

for all $k, h \in[0,1]$ and $\breve{u}, \check{c} \in \dot{C}([0,1], \mathbb{R})$, where

$$
\begin{aligned}
N(\breve{u}, \check{c})= & \eta_{1}[|\breve{u}(k)+\check{c}(k)|]+\eta_{2}[|\breve{u}(k)+\check{S} \breve{u}(k)|] \\
& +\eta_{3}[|\breve{u}(k)+\check{T} \check{c}(k)|] \\
& +\eta_{4} \frac{[|\breve{u}(k)+\check{S} \breve{u}(k)|]^{2} \cdot[|\check{c}(k)+\check{T} \check{c}(k)|]}{1+[|\breve{u}(k)+\check{c}(k)|]^{2}},
\end{aligned}
$$

where $\eta_{1}, \eta_{2}, \eta_{3}, \eta_{4} \geq 0$ and $\eta_{1}+\eta_{2}+2 \eta_{3}+\eta_{4}<1$. Then integral equations (71) have a solution.

Proof. By assumption (ii),

$$
\begin{aligned}
& |\check{S} \breve{u}(k)+\check{T} \check{c}(k)| \\
& \quad=\int_{0}^{k}\left|H_{1}\left(k, h, \breve{u}(h)+H_{2}(k, h, \check{c}(h))\right)\right| d h, \\
& \quad \leq \int_{0}^{k} \frac{\tau}{\left(\tau \sqrt{\|N(\breve{u}, \check{c})\|_{\tau}}+1\right)^{2}}\left([N(\breve{u}, \check{c})] e^{-\tau h}\right) e^{\tau h} d h, \\
& \quad \leq \int_{0}^{k} \frac{\tau}{\left(\tau \sqrt{\|N(\breve{u}, \check{c})\|_{\tau}}+1\right)^{2}}\|N(\breve{u}, \check{c})\|_{\tau} e^{\tau h} d h, \\
& \leq \frac{\tau\|N(\breve{u}, \check{c})\|_{\tau}}{\left(\tau \sqrt{\|N(\breve{u}, \check{c})\|_{\tau}}+1\right)^{2}} \int_{0}^{k} e^{\tau h} d h, \\
& \leq \frac{\|N(\breve{u}, \check{c})\|_{\tau}}{\left(\tau \sqrt{\|N(\breve{u}, \check{c})\|_{\tau}}+1\right)^{2}} e^{\tau k} .
\end{aligned}
$$

This implies that

$$
\begin{aligned}
|\check{S} \breve{u}(k)+\check{T} \check{c}(k)| e^{-\tau k} & \leq \frac{\|N(\breve{u}, \check{c})\|_{\tau}}{\left(\tau \sqrt{\|N(\breve{u}, \check{c})\|_{\tau}}+1\right)^{2}}, \\
\|\check{S} \breve{u}(k)+\check{T} \check{c}(k)\|_{\tau} & \leq \frac{\| N\left(\breve{u}, \check{c} \|_{\tau}\right.}{\left(\tau \sqrt{\|N(\check{u}, \check{c})\|_{\tau}}+1\right)^{2}}, \\
\frac{\tau \sqrt{\|N(\breve{u}, \check{c})\|_{\tau}}+1}{\sqrt{\|N(\breve{u}, \check{c})\|_{\tau}}} & \leq \frac{1}{\sqrt{\|\check{S} \breve{u}(k)+\check{T} \check{c}(k)\|_{\tau}}}, \\
\tau+\frac{1}{\sqrt{\|N(\breve{u}, \check{c})\|_{\tau}}} & \leq \frac{1}{\sqrt{\|\check{S} \breve{u}(k)+\check{T} \check{c}(k)\|_{\tau}}},
\end{aligned}
$$


which further implies that

$$
\tau-\frac{1}{\sqrt{\|\check{S} \check{u}(k)+\check{T} \check{c}(k)\|_{\tau}}} \leq \frac{-1}{\sqrt{\|N(\breve{u}, \check{c})\|_{\tau}}} .
$$

So all the conditions of Theorem 25 are satisfied for $(\check{c})=$ $-1 / \sqrt{\tilde{c}} ; \check{c}>0$; and $d_{\tau}(\breve{u}, \check{c})=\|\breve{u}+\check{c}\|_{\tau}$. Hence integral equations given in (71) have a unique common solution.

\section{Conflicts of Interest}

The authors declare that they have no conflicts of interest.

\section{Acknowledgments}

The authors acknowledge with thanks the Deanship of Scientific Research (DSR), King Abdulaziz University, Jeddah, that funded this article for financial support.

\section{References}

[1] S. Banach, "Sur les opérations dans les ensembles abstraits et leur application aux équations intégrales," Fundamenta Mathematicae, vol. 3, pp. 133-181, 1922.

[2] M. Abbas, B. Ali, and S. Romaguera, "Fixed and periodic points of generalized contractions in metric spaces," Fixed Point Theory and Applications, vol. 2013, article no. 243, 2013.

[3] Ö. Acar and I. Altun, "A fixed point theorem for multivalued mappings with $\delta$-Distance," Abstract and Applied Analysis, vol. 2014, Article ID 497092, 5 pages, 2014.

[4] Ö. Acar, G. Durmaz, and G. Minak, "Generalized multivalued F-contractions on complete metric spaces," Bull. Iranian Math. Society, vol. 40, no. 6, pp. 1469-1478, 2014.

[5] J. Ahmad, A. Al-Rawashdeh, and A. Azam, "New fixed point theorems for generalized F-contractions in complete metric spaces," Fixed Point Theory and Applications, vol. 2015, no. 1, article no. 80, 2015.

[6] M. Arshad, Z. Kadelburg, S. Radenović, A. Shoaib, and S. Shukla, "Fixed Points of $\alpha$-Dominated Mappings on Dislocated Quasi Metric Spaces," Filomat, vol. 31, no. 11, pp. 3041-3056, 2017.

[7] M. Arshad, A. Shoaib, and P. Vetro, "Common fixed points of a pair of hardy rogers type mappings on a closed ball in ordered dislocated metric spaces," journal of function spaces and applications, vol. 2013, Article ID 638181, 9 pages, 2013.

[8] M. Arshad, A. Shoaib, M. Abbas, and A. Azam, "Fixed points of a pair of Kannan type mappings on a closed ball in ordered partial metric spaces," Miskolc Mathematical Notes. A Publication of the University of Miskolc, vol. 14, no. 3, pp. 769784, 2013.

[9] M. Arshad, A. Azam, M. Abbas, and A. Shoaib, "Fixed points results of dominated mappings on a closed ball in ordered partial metric spaces without continuity," U.P.B. Scientific Bulletin. Series A. Applied Mathematics and Physics, vol. 76, no. 2, pp. 123134, 2014.

[10] M. Arshad, A. Shoaib, and I. Beg, "Fixed point of a pair of contractive dominated mappings on a closed ball in an ordered dislocated metric space," Fixed Point Theory and Applications, vol. 2013, article 115, 2013.
[11] E. Ameer and M. Arshad, “Two new generalization for F -contraction on closed ball and fixed point theorem with application," J. Mathematical Exten, vol. 11, pp. 1-24, 2017.

[12] M. Arshad, S. U. Khan, and J. Ahmad, "Fixed point results for Fcontractions involving some new rational expressions," Journal of Fixed Point Theory and Applications, vol. 11, no. 1, pp. 79-97, 2016.

[13] F. Bojor, "Fixed point theorems for Reich type contractions on metric spaces with a graph," Nonlinear Analysis. Theory, Methods \& Applications, vol. 75, no. 9, pp. 3895-3901, 2012.

[14] P. Hitzler and A. K. Seda, "Dislocated topologies," J. Elect. Engineering, vol. 51, no. (12/s), pp. 3-7, 2000.

[15] N. Hussain, J. Ahmad, and A. Azam, "On Suzuki-Wardowski type fixed point theorems," The Journal of Nonlinear Science and its Applications, vol. 8, no. 6, pp. 1095-1111, 2015.

[16] N. Hussain, S. Al-Mezel, and P. Salimi, "Fixed points for $\psi$ -graphic contractions with application to integral equations," Abstract and Applied Analysis, vol. 2013, Article ID 575869, 11 pages, 2013.

[17] N. Hussain and P. Salimi, "suzuki-wardowski type fixed point theorems for $\alpha$-GF-contractions," Taiwanese Journal of Mathematics, vol. 28, no. 20, pp. 1879-1895, 2014.

[18] A. Hussain, M. Arshad, and M. Nazim, "Connection of Ciric type F-contraction involving fixed point on closed ball," Ghazi. Uni. Journal of Sci, vol. 30, no. 1, pp. 283-291, 2017.

[19] A. Hussain, M. Arshad, and S. U. Khan, " $\tau$-Generalization of fixed point results for F-contraction," Bangmod Int. J. Math. Comp. Sci, vol. 1, no. 1, pp. 127-137, 2015.

[20] A. Hussain, M. Arshad, M. Nazam, and S. U. Khan, "New type of results involving closed ball with graphic contraction," Journal of Inequalities and Special Functions, vol. 7, no. 4, pp. 36-48, 2016.

[21] J. Jachymski, "The contraction principle for mappings on a metric space with a graph," Proceedings of the American Mathematical Society, vol. 136, no. 4, pp. 1359-1373, 2008.

[22] S. U. Khan, M. Arshad, A. Hussain, and M. Nazam, "Two new types of fixed point theorems for F-contraction," Journal of Advanced Studies in Topology, vol. 7, no. 4, pp. 251-260, 2016.

[23] J. Nadler, "Multi-valued contraction mappings," Pacific Journal of Mathematics, vol. 30, pp. 475-488, 1969.

[24] H. Piri and P. Kumam, "Some fixed point theorems concerning F-contraction in complete metric spaces," Fixed Point Theory and Applications, vol. 2014, no. 1, article no. 210, 2014.

[25] H. Piri, S. Rahrovi, H. Marasi, and P. Kumam, "Fixed point theorem for F-Khan-contractions on complete metric spaces and application to the integral equations," Journal of Nonlinear Sciences and Applications. JNSA, vol. 10, no. 9, pp. 4564-4573, 2017.

[26] N.-A. Secelean, "Iterated function systems consisting of Fcontractions," Fixed Point Theory and Applications, vol. 2013, article no. 277, 2013.

[27] M. Sgroi and C. Vetro, "Multi-valued F-contractions and the solution of certain functional and integral equations," Filomat, vol. 27, no. 7, pp. 1259-1268, 2013.

[28] A. Shoaib, “ $\alpha-\eta$ Dominated Mappings and Related Common Fixed Point Results in Closed Ball," Journal of Concrete and Applicable Mathematics, vol. 13, no. 1-2, pp. 152-170, 2015.

[29] A. Shoaib, A. Hussain, M. Arshad, and A. Azam, "Fixed point results for $\alpha^{\star}-\psi$-Ciric type multivalued mappings on an intersection of a closed ball and a sequence with graph," Journal of Mathematical Analysis, vol. 7, no. 3, pp. 41-50, 2016. 
[30] A. Shoaib, "Fixed Point Results for $\alpha^{\star}-\psi$-multivalued Mappings," Bulletin of Mathematical Analysis and Applications, vol. 8, no. 4, pp. 43-55, 2016.

[31] A. Shoaib, M. Arshad, and J. Ahmad, "Fixed point results of locally contractive mappings in ordered quasi-partial metric spaces," The Scientific World Journal, vol. 2013, Article ID 194897, 8 pages, 2013.

[32] J. Tiammee and S. Suantai, "Coincidence point theorems for graph-preserving multi-valued mappings," Fixed Point Theory and Applications, vol. 2014, article no. 70, 2014.

[33] D. Wardowski, "Fixed points of a new type of contractive mappings in complete metric spaces," Fixed Point Theory and Applications, vol. 2012, article no. 94, 2012. 


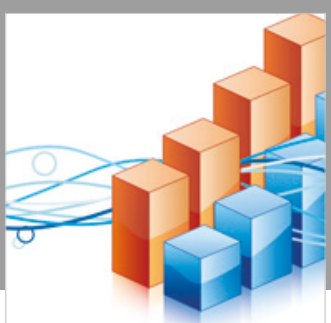

Advances in

Operations Research

\section{-n-m}
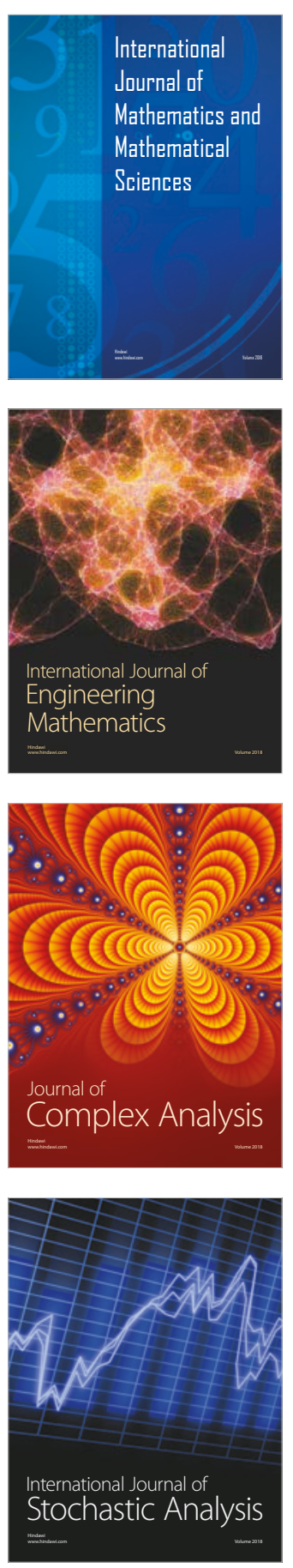
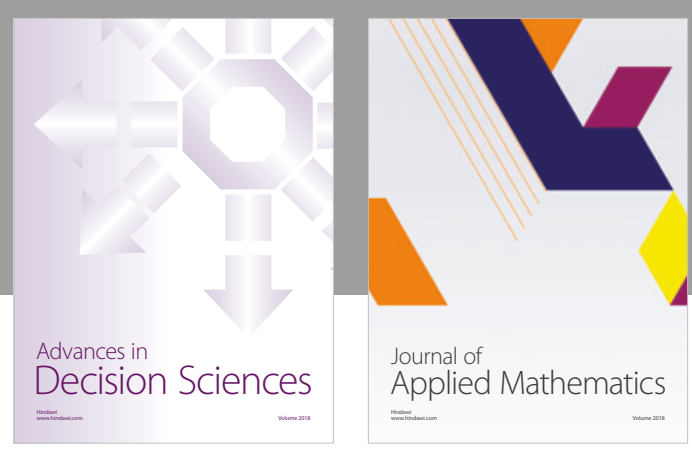

Journal of

Applied Mathematics
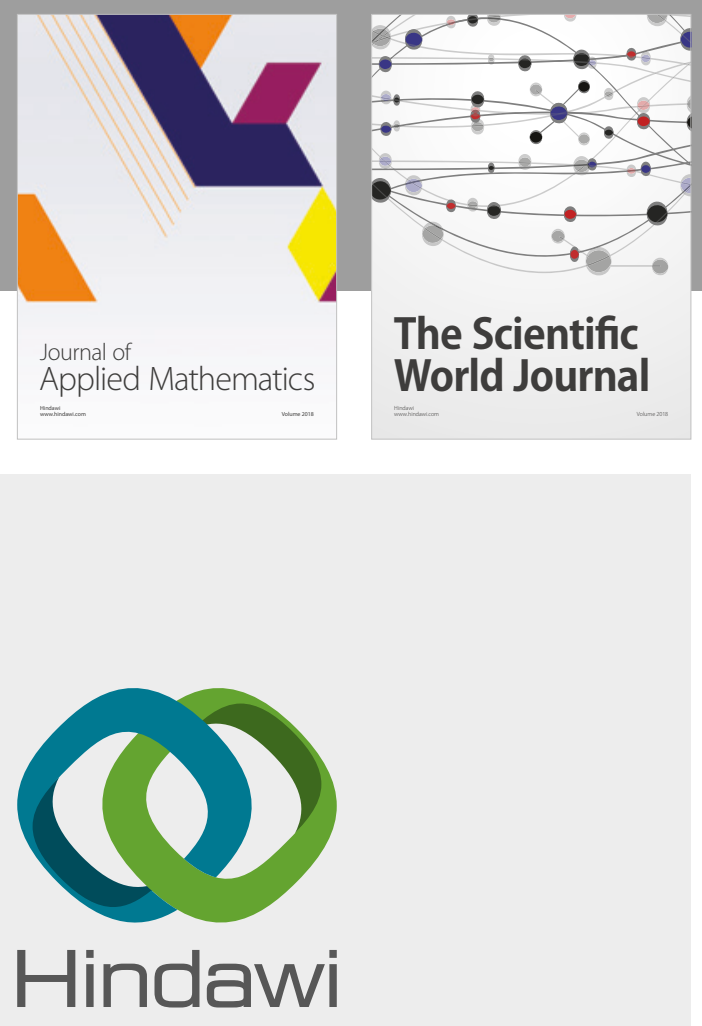

Submit your manuscripts at

www.hindawi.com

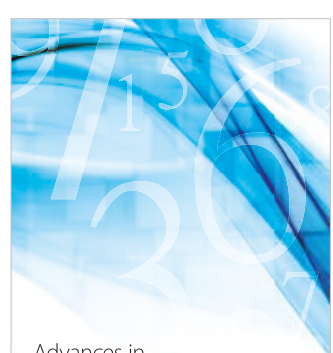

Advances in
Numerical Analysis
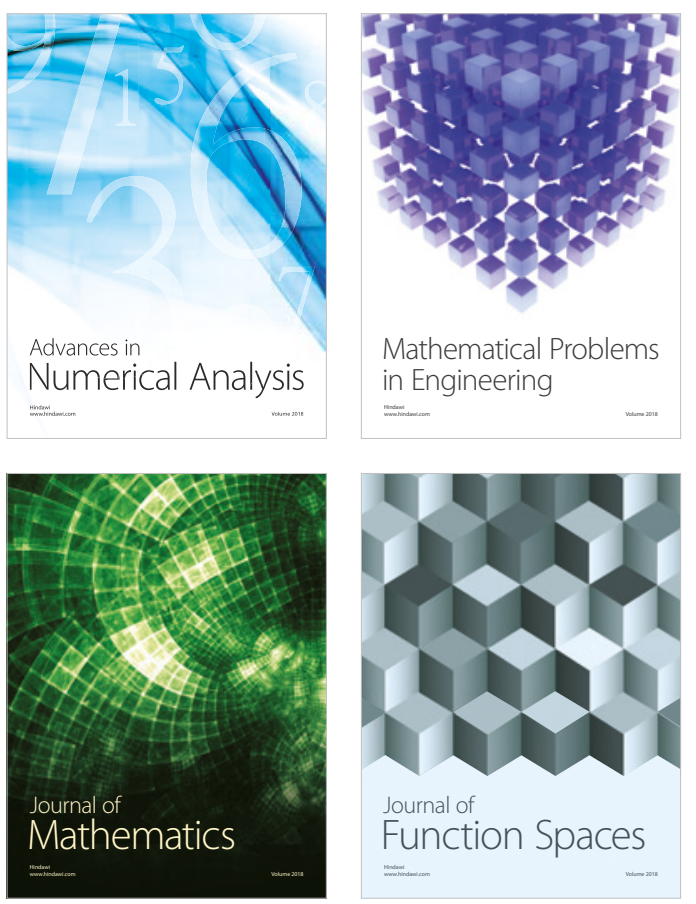

Mathematical Problems in Engineering

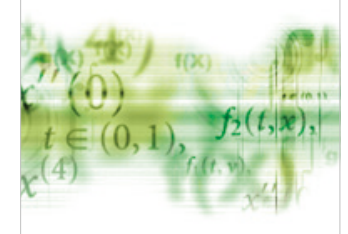

International Journal of

Differential Equations

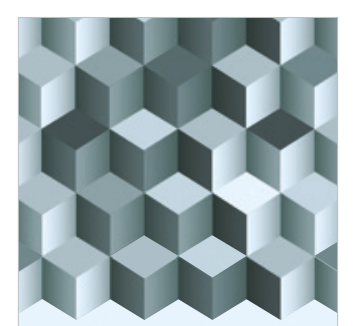

Journal of

Function Spaces

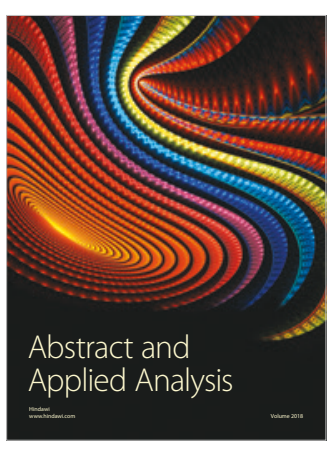

The Scientific

World Journal

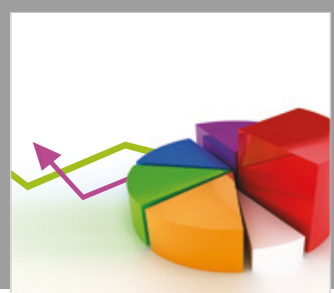

Journal of

Probability and Statistics
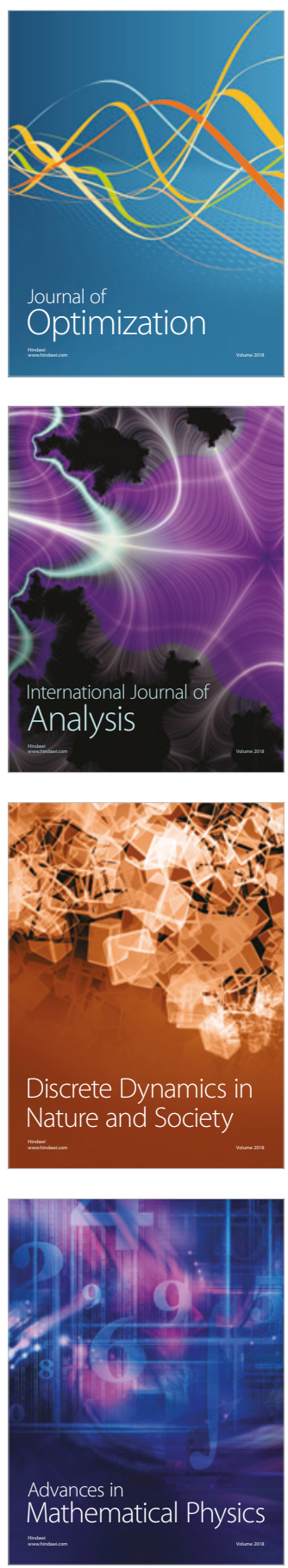